\title{
RECOVERING PLANE CURVES OF LOW DEGREE FROM THEIR INFLECTION LINES AND INFLECTION POINTS
}

\author{
BY \\ Marco PACINI* \\ Universidade Federal Fluminense \\ Rua M. S. Braga, Niterói (RJ) Brazil \\ e-mail:pacini@impa.br \\ AND \\ Damiano TeSta** \\ Mathematics Institute, University of Warwick \\ Coventry, CV4 7AL, United Kingdom \\ e-mail: adomani@gmail.com
}

\begin{abstract}
In this paper we consider the following problem: is it possible to recover a smooth plane curve of degree $d \geq 3$ from its inflection lines? We answer the posed question positively for a general smooth plane quartic curve, making the additional assumption that also one inflection point is given, and for any smooth plane cubic curve.
\end{abstract}

\section{Introduction}

In the last decade, several reconstruction theorems for plane and canonical curves defined over the field of complex numbers appeared in the literature. We mention the papers [CS1] and [L1, the first one showing that a general smooth plane quartic curve can be recovered from its 28 bitangents and the

* The first author was partially supported by CNPq, processo 300714/2010-6.

** The second author was partially supported by EPSRC grant number $\mathrm{EP} / \mathrm{F} 060661 / 1$.

Received March 7, 2011 and in revised form October 4, 2011 
second one generalizing the result to any smooth plane quartic. The result is extended in CS2 to a general smooth canonical curve and in GS to a general principally polarized abelian variety considering theta hyperplanes, the natural generalization of the bitangents of a plane quartic. Recently, an effective algorithm reconstructing a canonical curve from its theta hyperplanes has been obtained in L2].

In this paper we consider other distinguished lines associated to a plane curve. Recall that a smooth plane curve of degree $d \geq 3$ has $3 d(d-2)$ inflection lines, counted with multiplicity, i.e. lines cutting the curve in a point with multiplicity at least 3 , called inflection point of the curve. It is therefore natural to ask whether or not a plane curve of degree $d$ can be reconstructed from its inflections lines. Here we investigate the case of curves of degree at most 4, i.e. plane cubic curves and plane quartic curves.

The properties of the inflections points of plane curves have been widely studied by the classical geometers. For example, in [H1, H2, O. Hesse studied a pencil, also known as the Hesse pencil, given by the equation

$$
\lambda_{0}\left(x^{3}+y^{3}+z^{3}\right)-3 \lambda_{1} x y z=0
$$

where $\left[\lambda_{0}, \lambda_{1}\right] \in \mathbb{P}^{1}$ and $x, y, z$ are homogeneous coordinates on $\mathbb{P}^{2}$. The curves appearing in this pencil share the same inflection points (see also the proof of Theorem 4.2). For more details on the Hesse pencil, we refer the reader to [AD]. In [W], R. M. Winger considered curves of degree six sharing some inflection lines. More precisely, he introduced a pencil of plane curves of degree six with 12 common inflection points and 6 common inflection lines. But, to our knowledge, the problem of whether or not two distinct plane curves of degree $d$ could also share the whole set of inflection lines has never been considered, even for $d=3$, and therefore this question is still open.

The interest in reconstructing results for a smooth plane quartic curve consists also in the attempt to give an improved version of the classical Torelli Theorem for non-hyperelliptic curves of genus 3 . Indeed, let $C$ be a smooth plane quartic curve and $J(C)$ its Jacobian, with principal polarization $\Theta(C)$. Let $\Theta(C) \rightarrow\left(\mathbb{P}^{2}\right)^{\vee}$ be the Gauss map. If $C$ can be recovered by a finite set of its dual curve, then only a finite number of points in the image of the Gauss map are necessary to recover $C$. We refer to [ACGH, page 245] for more details on the Torelli map. 
For plane quartic curves, we need to make an additional assumption in the reconstruction result of Theorem 3.3 , which we can state as follows.

THEOREM 1: The general smooth plane quartic curve defined over the field of complex numbers is uniquely determined by its inflection lines and one inflection point. In other words, let $L \subset \mathbb{P}^{2}$ be a line and $p \in L$ a point. Let $X_{1}$ and $X_{2}$ be smooth plane quartic curves admitting $L$ as inflection line and $p$ as inflection point. If $X_{1}$ is sufficiently general, and if $X_{1}$ and $X_{2}$ have the same inflection lines, then $X_{1}=X_{2}$.

To prove Theorem 1, we use the same degeneration technique developed in CS1. Indeed, in Section 2, we study degenerations of inflection lines when a smooth quartic approaches a singular one, and in Lemma 3.2 we show that a nodal curve which is the union of a line and an irreducible cubic with a node is determined by limit inflection lines. Then we deduce the result for general smooth curves in Theorem 3.3. showing that a certain morphism is étale exactly as in CS1. In Lemma 3.2 we prove that the map associating to a smooth plane quartic its configuration of inflection lines is generically finite onto its image. Nevertheless, the following problem remains open

QUESTION: Is it possible to recover a smooth plane quartic curve only from its inflection lines?

For plane cubic curves, we can state our reconstruction result contained in Theorem 4.6 .

Theorem 2: Let $C \subset \mathbb{P}^{2}$ be a smooth plane cubic curve over a field $k$ of characteristic different from three and let $T_{C} \subset\left(\mathbb{P}^{2}\right)^{\vee}$ be the set of inflection lines of $C$. There is a unique (geometrically) integral curve $C^{\prime} \subset\left(\mathbb{P}^{2}\right)^{\vee}$ such that:

- if $\operatorname{char}(k) \neq 2$, then $C^{\prime}$ is a sextic with cusps at the points of $T_{C}$;

- if $\operatorname{char}(k)=2$ and $j(C) \neq 0$, then $C^{\prime}$ is a cubic containing $T_{C}$;

- if $\operatorname{char}(k)=2$ and $j(C)=0$, then $C^{\prime}$ is a cubic containing $T_{C}$, with vanishing $j$-invariant.

Moreover, the space of cubics in $\left(\mathbb{P}^{2}\right)^{\vee}$ containing $T_{C}$ has dimension one if and only if $(\operatorname{char}(k), j(C))=(2,0)$. In all cases, the curve $C^{\prime}$ described above is the dual of the curve $C$. 
The proof of this result proceeds by reconstructing the dual of the initial plane cubic. There are two configurations of nine points that are used in the argument: the nine inflection points in the plane containing the cubic and the nine inflection lines in the dual projective plane. The inflection lines are the base locus of a Halphen pencil of plane sextics of genus one. If the characteristic of the ground field is different from two, then we prove that an integral plane sextic with a singularity at one of these points is automatically singular at all the nine points and the singularities are all cuspidal. This cuspidal curve is the dual of the initial plane cubic and the reconstruction follows by projective duality. In this proof it is important that the dual of the plane cubic is a curve of degree six with cuspidal singularities at nine points, and can therefore be identified with a curve in the anti-bicanonical linear system of a blow up of the dual projective plane.

The results obtained in this paper may also be related to the results and questions of the paper [BKT] on the set-theoretic Torelli Theorem for curves over finite fields. Indeed, the inflection lines of a smooth plane cubic and the bitangent lines to a smooth plane quartic define effective cubic and square roots of the divisor class of a line in the plane. Similarly, the inflection lines to a plane quartic define cubic roots of pencils with a base point that are contained in the canonical system.

1.1. Notation. Throughout the paper we use the following notation and terminology. In Section 2 and Section 3, we work over $\mathbb{C}$. A curve is a projective, local complete intersection connected and reduced scheme of pure dimension 1. If $C$ is a curve, then $g_{C}:=1-\chi\left(\mathcal{O}_{C}\right)$ is the genus of $C$ and $\omega_{C}$ is its dualizing sheaf. Moreover, $C^{s m}$ is the smooth locus of $C$ and $C^{s i n g}:=C-C^{s m}$. If $D$ is a divisor of a curve $C$, we denote by $\nu_{p} D$ the multiplicity of $D$ at $p$, for a point $p \in C^{s m}$.

A nodal curve is a curve whose singular points are nodes. A subcurve $Z$ of a curve $C$ is a non-empty union of irreducible components of $C$ such that $Z \subsetneq C$. If $Z_{1}$ and $Z_{2}$ are subcurves of a curve $C$ with no common components and such that $Z_{1} \cap Z_{2}$ are nodes of $C$, we denote by $\Delta_{Z_{1} \cap Z_{2}}$ the Cartier divisor of $Z_{1}$ or $Z_{2}$ defined as $\Delta_{Z_{1} \cap Z_{2}}:=\sum_{p \in Z_{1} \cap Z_{2}} p$. If $Z$ is a subcurve of a curve $C$, we let $Z^{\prime}:=\overline{C-Z}$, and if $Z \cap Z^{\prime}$ are nodes of $C$, we let $\Delta_{Z}:=\Delta_{Z \cap Z^{\prime}}$. We say that a curve $C$ defined over a field $k$ has a cusp (respectively a tacnode) in a point $s \in C$ if the completion of the local ring of $C$ at $s$ is isomorphic 
to the quotient of the formal power series $\operatorname{ring} k[[x, y]]$ by the ideal $\left(y^{2}-x^{3}\right)$ (respectively $\left(y^{2}-x^{4}\right)$ ).

A family of curves is a proper and flat morphism $f: \mathcal{C} \rightarrow B$ whose fibers are curves. We denote by $\omega_{f}$ the relative dualizing sheaf of the family and $C_{b}:=f^{-1}(b)$, for $b \in B$. A smoothing of a curve $C$ is a family $f: \mathcal{C} \rightarrow B$, where $B$ is the spectrum of a discrete valuation ring with closed point 0 such that the general fiber is smooth and $C_{0}=f^{-1}(0)=C$. A regular smoothing of a curve $C$ is a smoothing $\mathcal{C} \rightarrow B$ of $C$ such that $\mathcal{C}$ is smooth everywhere except possibly at the points of $C$ which lie on exactly one irreducible component of $C$. If $\mathcal{L}$ is a line bundle on a curve $C$, we $\operatorname{set} \operatorname{deg}_{Z} \mathcal{L}:=\left.\operatorname{deg} \mathcal{L}\right|_{Z}$, for every subcurve $Z$ of $C$.

If $C$ is a plane curve, a smoothing of $C$ to plane curves is a smoothing $f: \mathcal{C} \rightarrow B$, where $\mathcal{C} \subset B \times \mathbb{P}^{2}$ and $f$ is the restriction of the projection onto the first factor. The dual curve $C^{\vee}$ of $C$ is defined as the closure in $\left(\mathbb{P}^{2}\right)^{\vee}$ of the set representing the tangents at the smooth points of $C$. It is worth observing that if $C$ is singular, then $C^{\vee}$ is not the flat limit of the duals of smooth curves approaching $C$ (see $\mathrm{K} 2$ for more information on the flat limit of dual curves). If $F(x, y, z)$ is a homogeneous polynomial in $x, y, z$, then $Z(F) \subseteq \mathbb{P}^{2}$ will denote its zero set.

If $V$ is a variety and $p$ is a point of $V$, we denote by $T_{p} V$ the tangent space to $V$ at $p$. If $S$ is a scheme, we denote by $S^{r e d}$ the reduced scheme associated to $S$.

\section{Limits of inflection lines}

Let $C$ be a plane quartic curve. If $C$ is irreducible, the Plücker formulas for plane curves provide the number of smooth inflection points of $C$. On the other hand, D. Eisenbud and J. Harris in EH1] consider the problem of determining what are the limits of Weierstrass points in families of curves degenerating to stable curves. So far, there are answers for curves of compact type, in [EH1, and for curves with at most two components, in [La, WL, EM]. But how do the inflection lines degenerate in a smoothing of $C$ ? For example, how many inflection lines degenerate to a line of the tangent cone at is a node of $C$, or to a line contained in $C$ ? In [K1], the author addresses the problem for pencils of type $\left\{G^{n}+t F\right\}_{t \in \mathbb{A}^{1}}$, where $n \geq 2$ and $F$ and $G$ are homogeneous polynomials 
with $\operatorname{deg} F=n \operatorname{deg} G$. In this section, we will give the complete list of limit inflection lines and their multiplicities for certain plane quartic curves.

Let $f: \mathcal{C} \rightarrow B$ be a regular smoothing of a curve $C$ whose irreducible components intersect at nodes (notice that $C$ need not be a nodal curve in the sense of Subsection [1.1, but it is a nodal curve in the sense of [EM]). Let $Z_{1}, \ldots, Z_{m}$ be the irreducible components of $C$ and assume that $g_{C}>0$. Following [EM, Definition 2.6], let $W^{*}$ be the Weierstrass subscheme of the generic fiber of $f$ and $W_{f}$ be the $f$-Weierstrass scheme, i.e. the closure of $W^{*}$ in $\mathcal{C}$. Consider the natural finite morphism $\psi_{f}: W_{f} \rightarrow B$. Set $W_{f, 0}:=W_{f} \cap C$ and let $\left[W_{f, 0}\right]$ be the associated cycle.

For every sheaf $\mathcal{M}=\omega_{f}\left(\sum_{j=1}^{m} t_{j} Z_{j}\right)$ and for each $i \in\{1, \ldots, m\}$, define $\rho_{\mathcal{M}, i}$ to be the natural map

$$
\rho_{\mathcal{M}, i}:\left.f_{*} \mathcal{M}\right|_{0} \rightarrow H^{0}\left(\left.\mathcal{M}\right|_{Z_{i}}\right) .
$$

By [EM, Theorem 2.2], there is a unique $m$-tuple of sheaves $\left(\mathcal{L}_{1}, \ldots, \mathcal{L}_{m}\right)$ on $\mathcal{C}$, where for each $i \in\{1, \ldots, m\}$ we have $\mathcal{L}_{i}=\omega_{f}\left(\sum_{j=1}^{m} t_{j} Z_{j}\right)$, for $t_{1}, \ldots, t_{m} \in \mathbb{Z}$, and such that the following conditions hold:

(i) the natural map $\rho_{\mathcal{L}_{i}, i}:\left.f_{*} \mathcal{L}_{i}\right|_{0} \rightarrow H^{0}\left(\left.\mathcal{L}_{i}\right|_{Z_{i}}\right)$ is injective;

(ii) the natural map $\rho_{\mathcal{L}_{i}, j}:\left.f_{*} \mathcal{L}_{i}\right|_{0} \rightarrow H^{0}\left(\left.\mathcal{L}_{i}\right|_{Z_{j}}\right)$ is not zero, if $j \neq i$.

Fix $i \in\{1, \ldots, m\}$. The sheaf $\mathcal{L}_{i}$ defined above is called the canonical sheaf of $f$ with focus on $Z_{i}$. Notice that a sheaf $\mathcal{M}=\omega_{f}\left(\sum_{j=1}^{m} t_{j} Z_{j}\right)$, for $t_{j} \in \mathbb{Z}$, is the canonical sheaf of $f$ with focus on $Z_{i}$ if, whenever $j \neq i$, the following conditions hold

$$
h^{0}\left(\left.\mathcal{M}\right|_{C}\right)=g, \quad h^{0}\left(\left.\mathcal{M}\right|_{Z_{i}^{\prime}}\left(-\Delta_{Z_{i}^{\prime}}\right)\right)=0 \quad \text { and } \quad h^{0}\left(\left.\mathcal{M}\right|_{Z_{j}^{\prime}}\left(-\Delta_{Z_{j}^{\prime}}\right)\right)<g .
$$

Indeed, for every $j \in\{1, \ldots, m\}$, consider the natural exact sequence

$$
\left.\left.\left.0 \rightarrow \mathcal{M}\right|_{Z_{j}^{\prime}}\left(-\Delta_{Z_{j}^{\prime}}\right) \rightarrow \mathcal{M}\right|_{C} \rightarrow \mathcal{M}\right|_{Z_{j}} \rightarrow 0 .
$$

In general, $\left.f_{*} \mathcal{M}\right|_{0}$ is a subspace of dimension $g$ of $H^{0}\left(\left.\mathcal{M}\right|_{C}\right)$. If the conditions in (11) hold, then $\left.f_{*} \mathcal{M}\right|_{0}=H^{0}\left(\left.\mathcal{M}\right|_{C}\right), \operatorname{Ker}\left(\rho_{\mathcal{M}, i}\right)=H^{0}\left(\left.\mathcal{M}\right|_{Z_{i}^{\prime}}\left(-\Delta_{Z_{i}^{\prime}}\right)\right)=0$ and $\operatorname{dim}\left(\operatorname{Ker}\left(\rho_{\mathcal{M}, j}\right)\right)=h^{0}\left(\left.\mathcal{M}\right|_{Z_{j}^{\prime}}\left(-\Delta_{Z_{j}^{\prime}}\right)\right)<g$ if $j \neq i$, from the long exact sequence in cohomology associated to (2). Thus, $\mathcal{M}$ is the canonical sheaf of $f$ with focus on $Z_{i}$.

If $\mathcal{L}_{i}$ is the canonical sheaf of $f$ with focus on $Z_{i}$, the limit canonical aspect of $f$ with focus on $Z_{i}$ is $\left(\operatorname{Im}\left(\rho_{\mathcal{L}_{i}, i}\right),\left.\mathcal{L}_{i}\right|_{Z_{i}}\right)$. Let $t_{i, 1}, \ldots, t_{i, m}$ be the unique 
integers such that $t_{i, i}=0$ and $\mathcal{L}_{i} \simeq \omega_{f}\left(\sum_{j=1}^{m} t_{i, j} Z_{j}\right)$. For every $p \in Z_{i} \cap Z_{j}$ such that $j \neq i$, we define the correction number for $\mathcal{L}_{i}$ at $p$ as $c_{Z_{i}}(p):=t_{i, j}$.

If $R_{Z_{i}}$ is the ramification divisor of the limit canonical aspect of $f$ with focus on $Z_{i}$, for every $i \in\{1, \ldots, m\}$, then by [EM, Theorem 2.8] we have

$$
\left[W_{f, 0}\right]=\sum_{i=1}^{m} R_{Z_{i}}+\sum_{p \in C^{\text {sing }}} c_{p} p
$$

where $c_{p}:=g\left(g-1-c_{Z_{i}}(p)-c_{Z_{j}}(p)\right)$, for $p \in Z_{i} \cap Z_{j}, i \neq j, i, j \in\{1, \ldots, m\}$.

If $\mathcal{L}$ is a line bundle on a smooth curve of genus $g$ and $R_{V}$ is the ramification divisor of a linear system $V \subset H^{0}(\mathcal{L})$ of dimension $r+1$, recall that by the Plücker formula we have

$$
\operatorname{deg} R_{V}=r(r+1)(g-1)+(r+1) \operatorname{deg} \mathcal{L} .
$$

Lemma 2.1: Let $f: \mathcal{C} \rightarrow B$ be a regular smoothing of a curve $C$ and $Z$ be an irreducible component of $C$ such that $Z \cap Z^{\prime}$ are nodes of $C$. Then $h^{0}\left(\left.\omega_{f}(Z)\right|_{C}\right)=$ $g_{C}$. Moreover, if $\# \Delta_{Z}=1$ and $g_{Z} \geq 1$, then $h^{0}\left(\left.\omega_{f}(2 Z)\right|_{C}\right)=g_{C}$.

Proof. Fix $i \in\{1,2\}$. By Riemann-Roch,

$$
h^{0}\left(\left.\omega_{f}(i Z)\right|_{C}\right)=g_{C}-1+h^{0}\left(\left.\mathcal{O}_{\mathcal{C}}(-i Z)\right|_{C}\right)
$$

Hence we only need to show that $h^{0}\left(\left.\mathcal{O}_{\mathcal{C}}(-i Z)\right|_{C}\right)=1$. Consider the natural exact sequence

$$
\left.\left.\left.0 \rightarrow \mathcal{O}_{\mathcal{C}}(-i Z)\right|_{Z}\left(-\Delta_{Z}\right) \rightarrow \mathcal{O}_{\mathcal{C}}(-i Z)\right|_{C} \rightarrow \mathcal{O}_{\mathcal{C}}(-i Z)\right|_{Z^{\prime}} \rightarrow 0
$$

Now, $h^{0}\left(\left.\mathcal{O}_{\mathcal{C}}(-i Z)\right|_{Z^{\prime}}\right)=h^{0}\left(\mathcal{O}_{Z^{\prime}}\left(-i \Delta_{Z^{\prime}}\right)\right)=0$. Thus, from the long exact sequence in cohomology, $h^{0}\left(\left.\mathcal{O}_{\mathcal{C}}(-i Z)\right|_{C}\right)=h^{0}\left(\left.\mathcal{O}_{\mathcal{C}}(-i Z)\right|_{Z}\left(-\Delta_{Z}\right)\right)$. If $i=1$, then $h^{0}\left(\left.\mathcal{O}_{\mathcal{C}}(-i Z)\right|_{Z}\left(-\Delta_{Z}\right)\right)=h^{0}\left(\mathcal{O}_{Z}\right)=1$, because $Z$ is connected. If $i=2$, $\Delta_{Z}=\{p\}$ and $g_{Z} \geq 1$, then $h^{0}\left(\left.\mathcal{O}_{\mathcal{C}}(-i Z)\right|_{Z}\left(-\Delta_{Z}\right)\right)=h^{0}\left(\mathcal{O}_{Z}(p)\right)=1$.

Although some parts of the following three lemmas are known to the specialists, we will provide complete proofs for the reader's convenience.

Lemma 2.2: Let $C$ be an irreducible plane quartic with a node $p$ and $Y$ be the normalization of $C$ at $p$. Let $g: \mathcal{C} \rightarrow B$ be a smoothing of $C$ to plane quartics. Assume that $\mathcal{C}$ has a singularity of $A_{1}$-type at $p$. Consider the blowup $\pi: \mathcal{X} \rightarrow \mathcal{C}$ of $\mathcal{C}$ at $p$, and set $f:=g \circ \pi$ and $X:=f^{-1}(0)=Y \cup E$, where $E \simeq \mathbb{P}^{1}$ is the exceptional component of $\pi$. If $R_{Y}$ is the ramification divisor of the limit 
canonical aspect of $f$ with focus on $Y$, then $\left[W_{f, 0}\right]=R_{Y}+3 p_{1}+3 p_{2}$, where $\left\{p_{1}, p_{2}\right\}:=E \cap Y$ and $\nu_{p_{i}} R_{Y} \in\{0,1\}$, for $i \in\{1,2\}$.

Proof. First of all, $\omega_{f}$ is the canonical sheaf of $f$ with focus on $Y$, because we have $h^{0}\left(\left.\omega_{f}\right|_{X}\right)=3, h^{0}\left(\left.\omega_{f}\right|_{Y^{\prime}}\left(-\Delta_{Y^{\prime}}\right)\right)=h^{0}\left(\mathcal{O}_{\mathbb{P}^{1}}\left(-p_{1}-p_{2}\right)\right)=0$, $h^{0}\left(\left.\omega_{f}\right|_{E^{\prime}}\left(-\Delta_{E^{\prime}}\right)\right)=h^{0}\left(\omega_{Y}\right)=2$, and hence the conditions listed in (1) hold. In particular, the correction number for $\omega_{f}$ at $p_{i}$ is $c_{Y}\left(p_{i}\right)=0$, for $i \in\{1,2\}$.

The morphism $\pi$ contracts $E$, hence $C=\left.\pi\right|_{Y}(Y)$. Moreover, $\pi^{*}\left(\mathcal{O}_{\mathcal{C}}(1)\right) \simeq$ $\pi^{*} \omega_{g} \simeq \omega_{f}$, because $g: \mathcal{C} \rightarrow B$ is a family of canonical curves. In this way, $\left.\pi\right|_{Y}$ is induced by a projective space of dimension two contained in the complete linear system $\left|\omega_{f} \otimes \mathcal{O}_{Y}\right|=\left|\omega_{Y}\left(p_{1}+p_{2}\right)\right|$. But $h^{0}\left(\omega_{Y}\left(p_{1}+p_{2}\right)\right)=3$, by Riemann-Roch, hence $\left.\pi\right|_{Y}$ is induced by $\left|\omega_{Y}\left(p_{1}+p_{2}\right)\right|$.

Set $\mathcal{L}:=\omega_{Y}\left(-p_{1}-p_{2}\right)$. We claim that $h^{0}(\mathcal{L})=0$. Indeed, by contradiction assume that $h^{0}(\mathcal{L}) \neq 0$. Then $\omega_{Y} \simeq \mathcal{O}_{Y}\left(p_{1}+p_{2}\right)$, because $Y$ is irreducible and $\mathcal{L}$ has degree zero. Thus, $h^{0}\left(\mathcal{O}_{Y}\left(p_{1}+p_{2}\right)\right)=2$ and $\omega_{Y}\left(p_{1}+p_{2}\right) \simeq \mathcal{O}_{Y}\left(p_{1}+p_{2}\right)^{\otimes 2}$. It follows that $\left.\pi\right|_{Y}$ factors via a degree 2 morphism $Y \rightarrow \mathbb{P}^{1}$, and hence $C=$ $\left.\pi\right|_{Y}(Y)$ is not a plane quartic, which is a contradiction.

If we set $\mathcal{L}_{E}:=\omega_{f}(Y)$, then the canonical sheaf of $f$ with focus on $E$ is $\mathcal{L}_{E}$, because $h^{0}\left(\left.\mathcal{L}_{E}\right|_{X}\right)=3$, by Lemma 2.1, $h^{0}\left(\left.\mathcal{L}_{E}\right|_{E^{\prime}}\left(-\Delta_{E^{\prime}}\right)\right)=h^{0}\left(\omega_{Y}\left(-p_{1}-p_{2}\right)\right)=$ 0 , by the above claim, and $h^{0}\left(\left.\mathcal{L}_{E}\right|_{Y^{\prime}}\left(-\Delta_{Y^{\prime}}\right)\right)=h^{0}\left(\mathcal{O}_{E}\right)=1$. In particular, the correction number for $\mathcal{L}_{E}$ at $p_{i}$ is $c_{E}\left(p_{i}\right)=1$, for $i \in\{1,2\}$.

Recall that $R_{Y}$ is the ramification divisor of $\left(V_{Y},\left.\omega_{f}\right|_{Y}\right)$, where $V_{Y}$ is the vector space $V_{Y}=\operatorname{Im}\left(H^{0}\left(\left.\omega_{f}\right|_{X}\right) \hookrightarrow H^{0}\left(\left.\omega_{f}\right|_{Y}\right)\right)$. We have $\left.\omega_{f}\right|_{Y} \simeq \omega_{Y}\left(p_{1}+p_{2}\right)$ and $\operatorname{dim} V_{Y}=h^{0}\left(\omega_{Y}\left(p_{1}+p_{2}\right)\right)=3$, hence $V_{Y}=H^{0}\left(\omega_{Y}\left(p_{1}+p_{2}\right)\right)$. Fix $i \in$ $\{1,2\}$. Notice that $V_{Y}\left(-p_{i}\right)=H^{0}\left(\omega_{Y}\left(p_{3-i}\right)\right)=H^{0}\left(\omega_{Y}\right) \simeq \mathbb{C}^{2}$. It follows that $\operatorname{dim} V_{Y}\left(-2 p_{i}\right)=h^{0}\left(\omega_{Y}\left(-p_{i}\right)\right)=1$, and hence $\nu_{p_{i}}\left(R_{Y}\right)=\operatorname{dim} V_{Y}\left(-3 p_{i}\right) \in$ $\{0,1\}$. Moreover, by (4), the ramification divisor of the limit canonical aspect of $f$ with focus on $E$ is $R_{E}=0$, hence by (3) we get

$$
\left[W_{f, 0}\right]=R_{Y}+3\left(2-c_{E}\left(p_{1}\right)\right) p_{1}+3\left(2-c_{E}\left(p_{2}\right)\right) p_{2}=R_{Y}+3\left(p_{1}+p_{2}\right) .
$$

Lemma 2.3: Let $C$ be an irreducible plane quartic with a cusp $p$ (respectively a tacnode $p$ ) and set $t:=8$ (respectively $t:=12$ ). Let $f: \mathcal{C} \rightarrow B$ be a smoothing of $C$ to plane quartics, with $\mathcal{C}$ smooth. Then there exists a finite base change $B^{\prime} \rightarrow B$ totally ramified over $0 \in B$, such that we have $\left[W_{f^{\prime}, 0}\right]=\sum_{i=1}^{24-t} q_{i}+t p$, where $p \notin\left\{q_{1}, \ldots, q_{24-t}\right\}$ and $f^{\prime}: \mathcal{C} \times{ }_{B} B^{\prime} \rightarrow B^{\prime}$ is the second projection morphism. 
Proof. As explained in [HM, Section $3 \mathrm{C}]$, there is a finite base change $B^{\prime} \rightarrow B$ totally ramified over $0 \in B$ and a family of curves $g: \mathcal{X} \rightarrow B^{\prime}$ such that, if $f^{\prime}: \mathcal{C}^{\prime}:=\mathcal{C} \times{ }_{B} B^{\prime} \rightarrow B^{\prime}$ is the second projection morphism, then $\mathcal{X}$ and $\mathcal{C}^{\prime}$ are $B^{\prime}$-isomorphic away from the central fibers and $X:=g^{-1}(0)=Y \cup E$, where $Y$ is the normalization of $C$ at $p$ and $E$ is a smooth connected curve of genus $g_{E}=1$ intersecting transversally $Y$ at the points over $p \in C$. In particular, $\#(Y \cap E)=1$, if $p$ is a cusp (respectively $\#(Y \cap E)=2$, if $p$ is a tacnode). Moreover, it follows from [P, Lemma 5.1] that, if $B^{\prime} \rightarrow B$ has degree 6 (respectively 4), then there exists a family $g: \mathcal{X} \rightarrow B^{\prime}$ as stated, with $\mathcal{X}$ smooth along $Y \cap E$ and with a $B^{\prime}$-morphism $\pi: \mathcal{X} \rightarrow \mathcal{C}^{\prime}$ which is an isomorphism away from the central fibers and contracting $E$ to $p$. In particular, it follows that $\left.\pi\right|_{Y}(Y)=C$. We are done if we show that $\left[W_{g, 0}\right]=\sum_{i=1}^{24-t} q_{i}+\sum_{i=1}^{t} q_{i}^{\prime}$, where $\left\{q_{1}, \ldots, q_{24-t}\right\} \subset Y-E$ and $\left\{q_{1}^{\prime}, \ldots, q_{t}^{\prime}\right\} \subset E$. Actually we will prove that $\left\{q_{1}^{\prime}, \ldots, q_{t}^{\prime}\right\} \subset E-Y$.

Set $\mathcal{L}_{Y}:=\omega_{g}(E)$. We claim that $\mathcal{L}_{Y}$ is the canonical sheaf of $g$ with focus on $Y$. Indeed, $h^{0}\left(\left.\mathcal{L}_{Y}\right|_{X}\right)=3$, by Lemma 2.1, $h^{0}\left(\left.\mathcal{L}_{Y}\right|_{Y^{\prime}}\left(-\Delta_{Y^{\prime}}\right)\right)=$ $h^{0}\left(\mathcal{O}_{E}\left(-\Delta_{E}\right)\right)=0, h^{0}\left(\left.\mathcal{L}_{Y}\right|_{E^{\prime}}\left(-\Delta_{E^{\prime}}\right)\right)=h^{0}\left(\omega_{Y}\left(\Delta_{Y}\right)\right)=2$, by Riemann-Roch. In particular, for every $r \in Y \cap E$, the correction number for $\mathcal{L}_{Y}$ at $r$ is $c_{Y}(r)=1$.

Assume that $p$ is a tacnode and set $\left\{r_{1}, r_{2}\right\}:=Y \cap E$. Arguing as before, $\mathcal{L}_{E}:=\omega_{g}(Y)$ is the canonical sheaf of $g$ with focus on $E$. In particular, the correction number for $\mathcal{L}_{E}$ at $r_{i}$ is $c_{E}\left(r_{i}\right)=1$, for $i \in\{1,2\}$. Recall that $R_{Y}$ is the ramification divisor of $\left(V_{Y},\left.\mathcal{L}_{Y}\right|_{Y}\right)$, where $V_{Y}$ is the vector space $V_{Y}=\operatorname{Im}\left(\rho_{Y}: H^{0}\left(\left.\mathcal{L}_{Y}\right|_{X}\right) \hookrightarrow H^{0}\left(\left.\mathcal{L}_{Y}\right|_{Y}\right)\right), \rho_{Y}(s)=\left.s\right|_{Y}$. Assume that $s^{\prime}\left(r_{i}\right)=0$, for $i \in\{1,2\}$, where $s^{\prime}=\rho_{Y}(s) \in V_{Y}$. Then $\left.s\right|_{E}=0$, because $\left.\mathcal{L}_{Y}\right|_{E} \simeq \mathcal{O}_{E}$ and hence

$$
V_{Y}\left(-r_{i}\right) \subseteq \rho_{Y}\left(H^{0}\left(\left.\mathcal{L}_{Y}\right|_{X}\left(-r_{1}-r_{2}\right)\right)\right)=H^{0}\left(\mathcal{O}_{Y}\left(r_{1}+r_{2}\right)\right)=\mathbb{C}^{2} .
$$

The other inclusion in (5) is clear, hence $\operatorname{dim} V_{Y}\left(-r_{i}\right)=2, \operatorname{dim} V_{Y}\left(-2 r_{i}\right)=$ $h^{0}\left(\mathcal{O}_{Y}\left(r_{3-i}\right)\right)=1$ and $\operatorname{dim} V_{Y}\left(-3 r_{i}\right)=h^{0}\left(\mathcal{O}_{Y}\left(r_{3-i}-r_{i}\right)\right)=0$, because $g_{Y}=1$. In this way, $\nu_{r_{i}} R_{Y}=0$, for $i \in\{1,2\}$. By (4), we have $\operatorname{deg} R_{Y}=12$, hence $R_{Y}=\sum_{i=1}^{12} q_{i}$, where $\left\{q_{1}, \ldots, q_{12}\right\} \subset Y-E$. Arguing similarly, if $R_{E}$ is the ramification divisor of the limit canonical aspect of $g$ with focus on $E$, we have $R_{E}=\sum_{i=1}^{12} q_{i}^{\prime}$, where $\left\{q_{1}^{\prime}, \ldots, q_{12}^{\prime}\right\} \subset E-Y$. It follows from (3) that $\left[W_{g, 0}\right]=R_{Y}+R_{E}$, and hence we are done if $p$ is a tacnode.

From now on, assume that $p$ is a cusp and set $r:=Y \cap E$. Recall that $C=$ $\left.\pi\right|_{Y}(Y)$. Moreover, we have $\pi^{*}\left(\mathcal{O}_{\mathcal{C}^{\prime}}(1)\right) \simeq \pi^{*} \omega_{f^{\prime}}$, because $f^{\prime}: \mathcal{C}^{\prime} \rightarrow B^{\prime}$ is a family 
of canonical curves, hence $\left.\pi^{*}\left(\mathcal{O}_{\mathcal{C}^{\prime}}(1)\right) \simeq \omega_{g} \otimes \mathcal{O}_{\mathcal{X}}(D)\right|_{X}$, for some Cartier divisor $D$ of $\mathcal{X}$ of type $D=a Y+b E$, for $a, b \in \mathbb{Z}$. But $\operatorname{deg}_{E} \pi^{*}\left(\mathcal{O}_{\mathcal{C}^{\prime}}(1)\right)=0$, because $\pi$ contracts $E$, and $\operatorname{deg}_{E} \omega_{g}=1$, hence necessarily $\left.\left.\mathcal{O}_{\mathcal{X}}(D)\right|_{X} \simeq \mathcal{O}_{\mathcal{X}}(E)\right|_{X}$. In this way, $\left.\pi\right|_{Y}$ is induced by a projective space of dimension two contained in $\left|\omega_{g}(E) \otimes \mathcal{O}_{Y}\right|=\left|\omega_{Y}(2 r)\right|$. But $h^{0}\left(\omega_{Y}(2 r)\right)=3$, by Riemann-Roch, hence $\left.\pi\right|_{Y}$ is induced by $\left|\omega_{Y}(2 r)\right|$.

Set $\mathcal{L}:=\omega_{Y}(-2 r)$. We claim that $h^{0}(\mathcal{L})=0$. Indeed, suppose by contradiction that $h^{0}(\mathcal{L}) \neq 0$. Then $\omega_{Y} \simeq \mathcal{O}_{Y}(2 r)$, because $Y$ is irreducible and $\mathcal{L}$ has degree zero, implying $h^{0}\left(\mathcal{O}_{Y}(2 r)\right)=2$ and $\omega_{Y}(2 r) \simeq \mathcal{O}_{Y}(2 r)^{\otimes 2}$. It follows that $\left.\pi\right|_{Y}$ factors via a degree 2 morphism $Y \rightarrow \mathbb{P}^{1}$, hence $C=\left.\pi\right|_{Y}(Y)$ is not a plane quartic, which is a contradiction.

Set $\mathcal{L}_{E}:=\omega_{g}(2 Y)$. We claim that $\mathcal{L}_{E}$ is the canonical sheaf of $g$ with focus on $E$. Indeed, $h^{0}\left(\left.\mathcal{L}_{E}\right|_{X}\right)=3$, by Lemma 2.1, $h^{0}\left(\left.\mathcal{L}_{E}\right|_{E^{\prime}}\left(-\Delta_{E^{\prime}}\right)\right)=h^{0}\left(\omega_{Y}(-2 r)\right)=$ 0 , by the above claim, and $h^{0}\left(\left.\mathcal{L}_{E}\right|_{Y^{\prime}}\left(-\Delta_{Y^{\prime}}\right)\right)=h^{0}\left(\mathcal{O}_{E}(2 r)\right)=2$, by RiemannRoch. In particular, the correction number for $\mathcal{L}_{E}$ at $r$ is $c_{E}(r)=2$.

Recall that $R_{Y}$ is the ramification divisor of $\left(V_{Y},\left.\mathcal{L}_{Y}\right|_{Y}\right)$, where $V_{Y}$ is the vector space $V_{Y}=\operatorname{Im}\left(H^{0}\left(\left.\mathcal{L}_{Y}\right|_{X}\right) \hookrightarrow H^{0}\left(\left.\mathcal{L}_{Y}\right|_{Y}\right)\right)$. Notice that $h^{0}\left(\left.\mathcal{L}_{Y}\right|_{Y}\right)=$ $h^{0}\left(\omega_{Y}(2 r)\right)=3$, hence $V_{Y}=H^{0}\left(\omega_{Y}(2 r)\right)$. We have $\operatorname{dim} V_{Y}(-r)=h^{0}\left(\omega_{Y}(r)\right)=$ $2, \operatorname{dim} V_{Y}(-2 r)=h^{0}\left(\omega_{Y}\right)=2, \operatorname{dim} V_{Y}(-3 r)=h^{0}\left(\omega_{Y}(-r)\right)=1$ and $\operatorname{dim} V_{Y}(-4 r)=h^{0}\left(\omega_{Y}(-2 r)\right)=0$. In this way, $\nu_{r} R_{Y}=2$, and we can write $R_{Y}=2 r+\sum_{i=1}^{\operatorname{deg} R_{Y}-2} q_{i}$, where $\left\{q_{1}, \ldots, q_{\operatorname{deg} R_{Y}-2}\right\} \subset Y-E$.

Recall that $R_{E}$ is the ramification of $\left(V_{E},\left.\mathcal{L}_{E}\right|_{E}\right)$, where $V_{E}$ is the vector space $V_{E}=\operatorname{Im}\left(H^{0}\left(\left.\mathcal{L}_{E}\right|_{X}\right) \hookrightarrow H^{0}\left(\left.\mathcal{L}_{E}\right|_{E}\right)\right)$. Notice that $h^{0}\left(\left.\mathcal{L}_{E}\right|_{E}\right)=H^{0}\left(\mathcal{O}_{E}(3 r)\right)=3$, hence $V_{E}=H^{0}\left(\mathcal{O}_{E}(3 r)\right)$. We have $\operatorname{dim} V_{E}(-r)=h^{0}\left(\mathcal{O}_{E}(2 r)\right)=2$, $\operatorname{dim} V_{E}(-2 r)=h^{0}\left(\mathcal{O}_{E}(r)\right)=1, \operatorname{dim} V_{E}(-3 r)=h^{0}\left(\mathcal{O}_{E}\right)=1$ and $\operatorname{dim} V_{E}(-4 r)=h^{0}\left(\mathcal{O}_{E}(-r)\right)=0$. In this way, $\nu_{r} R_{E}=1$, and we can write $R_{E}=r+\sum_{i=1}^{\operatorname{deg} R_{E}-1} q_{i}^{\prime}$, where $\left\{q_{1}^{\prime}, \ldots, q_{\operatorname{deg} R_{E}-1}^{\prime}\right\} \subset E-Y$. By (4), $\operatorname{deg} R_{E}=9$, hence (3) implies that $\left[W_{g, 0}\right]=R_{Y}+R_{E}-3 r=\sum_{i=1}^{16} q_{i}+\sum_{i=1}^{8} q_{i}^{\prime}$, and we are done if $p$ is a cusp.

Lemma 2.4: Let $C$ be a nodal plane quartic curve which is the union of a smooth irreducible cubic $Y$ and a line $Z$, and set $\left\{p_{1}, p_{2}, p_{3}\right\}:=Y \cap Z$. If $f: \mathcal{C} \rightarrow B$ is a regular smoothing of $C$, then

$$
\left[W_{f, 0}\right]=R+\left(s_{1}+\cdots+s_{6}\right)+3\left(p_{1}+p_{2}+p_{3}\right)
$$

where $\left\{s_{1}, \ldots, s_{6}\right\} \subset Z \cap C^{s m}$ and $R$ is the ramification divisor of $\left|\mathcal{O}_{Y}(1)\right|$. 
Proof. Set $\mathcal{L}_{Y}:=\omega_{f}$. The canonical sheaf of $f$ with focus on $Y$ is $\mathcal{L}_{Y}$, because $h^{0}\left(\left.\mathcal{L}_{Y}\right|_{C}\right)=3, h^{0}\left(\left.\mathcal{L}_{Y^{\prime}}\right|_{Y^{\prime}}\left(-\Delta_{Y^{\prime}}\right)\right)=h^{0}\left(\omega_{Z}\right)=0$, and $h^{0}\left(\left.\mathcal{L}_{Y}\right|_{Z^{\prime}}\left(-\Delta_{Z^{\prime}}\right)\right)=$ $h^{0}\left(\omega_{Y}\right)=1$. In particular, the correction number for $\mathcal{L}_{Y}$ at $p_{i}$ is $c_{Y}\left(p_{i}\right)=0$, for $i \in\{1,2,3\}$. Set $\mathcal{L}_{Z}:=\omega_{f}(Y)$. The canonical sheaf of $f$ with focus on $Z$ is $\mathcal{L}_{Z}$, because $h^{0}\left(\left.\mathcal{L}_{Z}\right|_{C}\right)=3$, by Lemma 2.1, $h^{0}\left(\left.\mathcal{L}_{Z}\right|_{Z^{\prime}}\left(-\Delta_{Z^{\prime}}\right)\right)=h^{0}\left(\omega_{Y}\left(-\Delta_{Y}\right)\right)=0$ and $h^{0}\left(\left.\mathcal{L}_{Z}\right|_{Y^{\prime}}\left(-\Delta_{Y^{\prime}}\right)\right)=h^{0}\left(\omega_{Z}\left(\Delta_{Z}\right)\right)=2$. In particular, the correction number for $\mathcal{L}_{Z}$ at $p_{i}$ is $c_{Z}\left(p_{i}\right)=1$, for $i \in\{1,2,3\}$. If $R_{Z}$ is the ramification divisor of the limit canonical aspect of $f$ with focus on $Z$, then $\operatorname{deg}\left(R_{Z}\right)=6$, by (4). Hence, by (3), we are done if we show that $\nu_{p_{i}} R_{Z}=0$, for $i \in\{1,2,3\}$.

Consider $V_{Z}=\operatorname{Im}\left(\rho_{Z}: H^{0}\left(\left.\mathcal{L}_{Z}\right|_{C}\right) \hookrightarrow H^{0}\left(\left.\mathcal{L}_{Z}\right|_{Z}\right)\right), \rho_{Z}(s)=\left.s\right|_{Z}$. Assume that $s^{\prime}\left(p_{1}\right)=0$, for some $s^{\prime}=\rho_{Z}(s) \in V_{Z}$. Then $\left.s\right|_{Y}=0$, because $\left.\mathcal{L}_{Z}\right|_{Y} \simeq \mathcal{O}_{Y}$, and hence

(6) $V_{Z}\left(-p_{1}\right) \subseteq \rho_{Z}\left(H^{0}\left(\left.\mathcal{L}_{Z}\right|_{C}\left(-p_{1}-p_{2}-p_{3}\right)\right)\right)=H^{0}\left(\omega_{Z}\left(p_{1}+p_{2}+p_{3}\right)\right)=\mathbb{C}^{2}$.

Since the other inclusion in (6) is clear, we get $\operatorname{dim} V_{Z}\left(-p_{1}\right)=2$, and hence also $\operatorname{dim} V_{Z}\left(-2 p_{1}\right)=1$ and $\operatorname{dim} V_{Z}\left(-3 p_{1}\right)=0$. In this way, $\nu_{p_{1}} R_{Z}=0$, and similarly $\nu_{p_{2}} R_{Z}=\nu_{p_{3}} R_{Z}=0$, and hence we are done.

Let $\mathbb{P}^{14}$ be the projective space parameterizing plane quartic curves and $[C] \in \mathbb{P}^{14}$ be the point parameterizing a plane quartic $C$. Let $\mathcal{V} \subset \mathbb{P}^{14}$ be the open subset parameterizing reduced quartic curves that are GIT-semistable with respect to the natural action of $P G L(3)$ on $\mathbb{P}^{14}$ and with finite stabilizer. If $[C] \in \mathcal{V}$, then $C$ is reduced and a singular point of $C$ is a node, a cusp or a tacnode. The double conics are the unique GIT-semistable non-reduced quartics and if $C$ is a smooth quartic, then $[C] \in \mathcal{V}$. We refer to MFK, Chapter 4.2], [CS1, Section 3.4] or [AF for a detailed list of the curves parameterized by $\mathcal{V}$.

If $C$ is a smooth plane curve, an inflection line of $C$ is a line cutting $C$ in a point with multiplicity at least 3 . Recall that a smooth plane quartic admits exactly 24 inflection lines, counted with multiplicity. We denote by $\mathcal{F}_{C} \in \operatorname{Sym}^{24}\left(\mathbb{P}^{2 \vee}\right)$ the configuration of inflection lines of a smooth plane quartic curve $C$. Let $\mathcal{V}^{0} \subset \mathcal{V}$ be the open subset parameterizing smooth plane quartics and consider the morphism $\mathcal{F}^{*}: \mathcal{V}^{0} \rightarrow \operatorname{Sym}^{24}\left(\mathbb{P}^{2}\right)$ such that $\mathcal{F}^{*}([C])=\mathcal{F}_{C}$, for every $[C] \in \mathcal{V}^{0}$.

Proposition 2.5: There exists a morphism $\mathcal{F}: \mathcal{V} \rightarrow \operatorname{Sym}^{24}\left(\mathbb{P}^{2}\right)$ such that $\left.\mathcal{F}\right|_{\mathcal{V}^{0}}=\mathcal{F}^{*}$. 
Proof. Let $\Gamma$ be the closure inside $\mathcal{V} \times \operatorname{Sym}^{24}\left(\mathbb{P}^{2^{\vee}}\right)$ of the graph

$$
\Gamma^{0}:=\left\{\left([C], \mathcal{F}_{C}\right):[C] \in \mathcal{V}^{0}\right\} \subset \mathcal{V} \times \operatorname{Sym}^{24}\left(\mathbb{P}^{2 \vee}\right)
$$

Let $\pi_{1}: \Gamma \rightarrow \mathcal{V}$ and $\pi_{2}: \Gamma \rightarrow \operatorname{Sym}^{24}\left(\mathbb{P}^{2} \vee\right)$ be respectively the restriction of the first and the second projection morphism.

If $[C] \in \mathcal{V}$, we claim that only a finite number of lines can be limits of inflection lines of smooth curves degenerating to $C$. In fact, recall that $C$ is reduced and its singularities are double points. Consider a smoothing of $C$, and let $L$ be a line which is a limit of inflection lines of the general curve of the family. It follows that $L$ cuts $C$ with multiplicity at least 3 in a point $p$. If $L$ is a linear component of $C$, then it varies in a finite set. Suppose that $L \not \subset C$. If $p \in C^{\text {sing }}$, then $p$ is a double point of $C$, and $L$ is one of the lines of the tangent cone of $C$ at $p$. In particular, $L$ varies in a finite set. If $p \in C^{s m}$, then $L$ corresponds to a singular point of the dual curve $C^{\vee}$, hence $L$ varies again in a finite set, because we work over the field of complex numbers, and hence $C^{\vee}$ is reduced.

In particular, the fibers of $\pi_{1}$ are finite. Notice that $\mathcal{V}$ is smooth, because it is an open subset of $\mathbb{P}^{14}$. Moreover, $\left.\pi_{1}\right|_{\Gamma^{0}}$ is an isomorphism onto the smooth variety $\mathcal{V}^{0}$, thus $\Gamma^{0}$, and hence $\Gamma$, are irreducible. Since $\pi_{1}$ is a birational morphism, $\pi_{1}$ is an isomorphism, by [G, Corollaire 4.4.9]. The required morphism is $\mathcal{F}:=\pi_{2} \circ \pi_{1}^{-1}$.

Remark 2.6: In the proof of Proposition 2.5 we did not use the condition that the points of $\mathcal{V}$ parametrize a GIT-semistable curve. Indeed, the same proof shows that $\mathcal{F}^{*}$ actually extends to the open subset of $\mathbb{P}^{14}$ parametrizing reduced quartics with singular points of multiplicity two.

For every $[C] \in \mathcal{V}$, set $\mathcal{F}_{C}:=\mathcal{F}([C]) \in \operatorname{Sym}^{24}\left(\mathbb{P}^{2^{\vee}}\right)$. We can view $\mathcal{F}_{C}$ as a (possibly non-reduced) hypersurface of $\mathbb{P}^{2}$. We call a line $L \subset \mathbb{P}^{2}$ an inflection line of $C$ if $L$ is a component of $\mathcal{F}_{C}^{r e d}$. If $L$ is not a component of $\mathcal{F}_{C}$, we set $\mu_{L} \mathcal{F}_{C}=0$, otherwise $\mu_{L} \mathcal{F}_{C}$ will be the multiplicity of $L$ as a component of $\mathcal{F}_{C}$. For every $L \subset \mathcal{F}_{C}^{r e d}$, we say that $L$ is of type $\mathbf{0}$ if $L \cap C \subset C^{s m}$, of type $\mathbf{1}$ if $L \cap C^{\text {sing }} \neq \emptyset$ and $L \not \subset C$, and degenerate if $L \subset C$.

Remark 2.7: To compute the multiplicity of a component of $\mathcal{F}_{C}$, for $[C] \in \mathcal{V}$, we will use the following observation. Consider the incidence variety

$$
\mathcal{I}:=\left\{([C], L): L \subseteq \mathcal{F}_{C}\right\} \subseteq \mathcal{V} \times\left(\mathbb{P}^{2}\right)^{\vee}
$$


and the finite morphism $\pi_{\mathcal{I}}: \mathcal{I} \rightarrow \mathcal{V}$ of degree 24 obtained by restricting the first projection $\mathcal{V} \times\left(\mathbb{P}^{2}\right)^{\vee} \rightarrow \mathcal{V}$ to $\mathcal{I}$. The cycle associated to the fiber of $\pi_{\mathcal{I}}$ over $[C]$ is $\sum_{L \subseteq \mathcal{F}_{C}^{\text {red }}}\left(\mu_{L} \mathcal{F}_{C}\right) L$. Notice that if $\mathcal{C} \rightarrow B$ is a smoothing of $C$ to general smooth plane curves, with associated morphism $B \rightarrow \mathcal{V}$, and if we set $\mathcal{I}_{B}:=B \times \mathcal{V} \mathcal{I}$, then the first projection $\pi_{\mathcal{I}_{B}}: \mathcal{I}_{B} \rightarrow B$ is a finite morphism of degree 24 and the cycle associated to the fiber of $\pi_{\mathcal{I}_{B}}$ over $0 \in B$ is again $\sum_{L \subseteq \mathcal{F}_{C}^{\text {red }}}\left(\mu_{L} \mathcal{F}_{C}\right) L$. We would like to warn the reader of a possibly confusing point: while the limits of the inflection lines do not depend on the chosen smoothing, the limits of the inflection points may depend on the smoothing.

LEMMA 2.8: Let $B$ be the spectrum of a discrete valuation ring with closed point 0. Let $\gamma: C \rightarrow B$ be a finite morphism and $\nu: C^{\nu} \rightarrow C$ be the normalization of $C$. Let $\sum_{p \in \gamma^{-1}(0)} c_{p} p$ and $\sum_{(\gamma \circ \nu)^{-1}(0)} d_{q} q$ be the cycles associated to the fiber respectively of $\gamma$ and $\gamma \circ \nu$ over 0 , where $c_{p}$ and $d_{q}$ are positive integers. Then for every $p \in \gamma^{-1}(0)$ we have $c_{p}=\sum_{q \in \nu^{-1}(p)} d_{q}$.

Proof. If $p \in C^{s m}$, we have nothing to prove. If $p \in C^{\text {sing }}$, pick the normalization $\alpha: D \rightarrow C$ of $C$ at $p$. Then $\nu=\alpha \circ \beta$, where $\beta: C^{\nu} \rightarrow D$ is the normalization of $D$. Let $\sum_{r \in(\gamma \circ \alpha)^{-1}(0)} e_{r} r$ be the cycle associated to the fiber of $\gamma \circ \alpha$ over 0 , where $e_{r}$ is a positive integer. If $d$ is the degree of $\gamma$, then we have

$$
c_{p}=d-\sum_{p \neq p^{\prime} \in \gamma^{-1}(0)} c_{p^{\prime}}=d-\sum_{\substack{r \in(\gamma \circ \alpha)^{-1}(0) \\ r \notin \alpha^{-1}(p)}} e_{r}=\sum_{r^{\prime} \in \alpha^{-1}(p)} e_{r^{\prime}}=\sum_{q \in \nu^{-1}(p)} d_{q}
$$

where the second equality follows because $\alpha$ is an isomorphism away from $\alpha^{-1}(p)$, and the fourth equality follows because $\beta$ is an isomorphism locally at any $q \in \beta^{-1}\left(\alpha^{-1}(p)\right)=\nu^{-1}(p)$.

Lemma 2.9: Let $f: \mathcal{C} \rightarrow B$ be a smoothing of an irreducible plane cubic $C$ with a node (respectively a cusp). Set $k=3$ (respectively $k=1$ ). Then exactly $k$ lines cutting $C$ in a smooth point with multiplicity 3 are degenerations of inflection lines of the general fiber of $f$.

Proof. Let $h$ be the number of lines cutting $C$ in a smooth point with multiplicity 3. By [EH2, pages 153-154], any such line is a degeneration of an inflection line of the general fiber of $f$, hence we are done if we show that $h=k$. The dual 
curve $C^{\vee} \subset\left(\mathbb{P}^{2}\right)^{\vee}$ of $C$ is irreducible and, since we work over the complex numbers, its singular locus consists exactly of $h$ cusps. It follows that $g_{C^{\vee}}-h=0$, because $C$ and $C^{\vee}$ are birational. Let $d$ be the degree of $C^{\vee}$. If $C$ has a node, then $d=4$ and hence $h=g_{C^{\vee}}=3$. If $C$ has a cusp, then $d=3$ and hence $h=g_{C^{\vee}}=1$.

Proposition 2.10: Let $[C] \in \mathcal{V}$ and $L \subset \mathbb{P}^{2}$ be a line. The line $L$ is an inflection line of $C$ if and only if either $L$ is contained in the tangent cone of $C$ at some singular point of $C$, or $L$ is an inflection line of type 0 of $C$. Moreover, assuming that $L$ is an inflection line of $C$, the multiplicity of $L$ in $\mathcal{F}_{C}$ satisfies the following conditions:

(i) if $L$ is a line of the tangent cone of $C$ at some $p \in C^{\text {sing }}$ such that either $L \not \subset C$ or $L \subset C$ and $L$ is tangent at $p$ to a component of $C$ different from $L$, then $\mu_{L} \mathcal{F}_{C} \in\{3,4\}, \mu_{L} \mathcal{F}_{C}=8, \mu_{L} \mathcal{F}_{C}=12$ if $p$ is respectively a node, a cusp, a tacnode;

(ii) if $L$ is a component of $C$ which is not tangent to any other component of $C$, then $\mu_{L} \mathcal{F}_{C}=6$;

(iii) if $L$ is an inflection line of type 0 of $C$, then $\mu_{L} \mathcal{F}_{C} \leq 2$, and if equality holds, then $C$ is irreducible.

Proof. Let $p$ be the limit of an inflection point in a fixed smoothing of $C$. Through the point $p$ there is a line $L$ in $\mathcal{F}_{C}$ meeting $C$ with multiplicity at least three at $p$. If $p \in C^{s m}$, then the line $L$ is an inflection line of type 0 , or $L$ is a component of $C$. If $p \in C^{\text {sing }}$, then the line $L$ is contained in the tangent cone to $C$ at $p$, since the point $p$ has multiplicity 2 in $C$ and at least 3 in $L \cap C$. This proves that the lines of $\mathcal{F}_{C}$ must be of the stated form.

We now prove the converse, establishing at the same time the results about the multiplicities. Let $g: \mathcal{C} \rightarrow B$ be a smoothing of $C=g^{-1}(0)$ to general plane quartics, and let $b: B \rightarrow \mathcal{V}$ be the associated morphism. Recall the varieties $\mathcal{I} \hookrightarrow \mathcal{V} \times\left(\mathbb{P}^{2}\right)^{\vee}$ and $\mathcal{I}_{B}=B \times \mathcal{V} \mathcal{I} \hookrightarrow B \times \mathcal{V} \mathcal{V} \times\left(\mathbb{P}^{2}\right)^{\vee} \simeq B \times\left(\mathbb{P}^{2}\right)^{\vee}$ defined in Remark 2.7 Consider the relative Gauss $B$-map $\theta: \mathcal{C} \rightarrow B \times\left(\mathbb{P}^{2}\right)^{\vee}$ defined as $\theta(q)=\left(g(q), T_{q} C_{g(q)}\right)$, where $T_{q} C_{g(q)}$ is the tangent line to $C_{g(q)}=g^{-1}(g(q))$ at $q$. Notice that $\theta$ is defined away from $C^{\operatorname{sing}}$. Consider the $g$-Weierstrass scheme $W_{g} \hookrightarrow \mathcal{C}$ and let $\psi_{g}: W_{g} \rightarrow B$ be the associated finite morphism of degree 24 . Let $\nu: W_{g}^{\nu} \rightarrow W_{g}$ be the normalization of $W_{g}$ and $\gamma: W_{g}^{\nu} \rightarrow B \times\left(\mathbb{P}^{2}\right)^{\vee}$ the morphism extending $\left.\theta\right|_{W_{g}}$. Notice that $\theta$ sends the points of $W_{g}$ away from 
$\psi_{g}^{-1}(0)$ to points of $\mathcal{I}_{B}$, hence $\gamma$ factors through the inclusion $\mathcal{I}_{B} \hookrightarrow B \times\left(\mathbb{P}^{2}\right)^{\vee}$. We get the following commutative diagram.

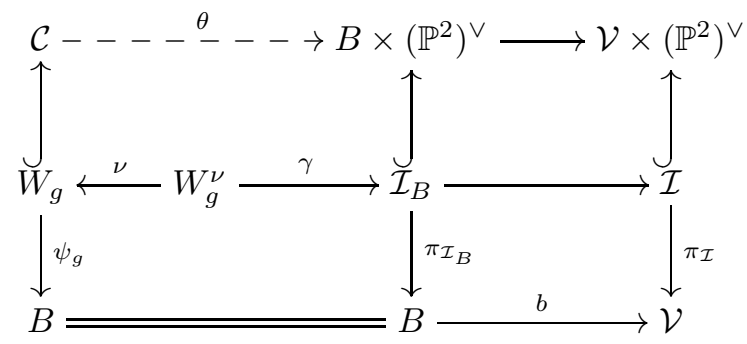

It follows from Remark 2.7 that $\gamma\left(\nu^{-1}(p)\right)$ parametrizes the set of lines of $\mathcal{F}_{C}$ cutting $C$ with multiplicity at least 3 in $p$, for every $p \in \psi_{g}^{-1}(0)$. In particular,

$$
\gamma\left(\nu^{-1}(p)\right) \cap \gamma\left(\nu^{-1}\left(p^{\prime}\right)\right)=\emptyset,
$$

for every $p, p^{\prime} \in \psi_{g}^{-1}(0)$ such that $p \neq p^{\prime}$ and such that the line through $p$ and $p^{\prime}$ is not contained in $C$.

Assume that $C$ is irreducible and that $L$ is a line of the tangent cone of $C$ at some $p_{0} \in C^{s i n g}$, so that $L \not \subset C$. Suppose that $p_{0}$ is a cusp or a tacnode. Choose a smoothing $g: \mathcal{C} \rightarrow B$ of $C$ with $\mathcal{C}$ smooth. Then there is a finite base change totally ramified over $0 \in B$ such that the statement of Lemma 2.3 holds. To avoid cumbersome notations, we keep the same symbols for the family obtained from $g: \mathcal{C} \rightarrow B$ after the base change. By Lemma 2.3. we have $\left[W_{g, 0}\right]=\sum q_{i}+t p_{0}$, where $p_{0} \notin\left\{q_{1}, \ldots, q_{24-t}\right\}$ and $t=8$, if $p_{0}$ is a cusp, and $t=12$, if $p_{0}$ is a tacnode. Observe that $p_{0} \in W_{g}$ and $L$ is the unique line cutting $C$ with multiplicity at least 3 at $p_{0}$, and hence $\gamma\left(\nu^{-1}\left(p_{0}\right)\right)=(0, L)$. Using (7), we deduce the following set-theoretic equality

$$
\nu^{-1}\left(p_{0}\right)=\gamma^{-1}((0, L)) .
$$

Since $\pi_{\mathcal{I}_{B}} \circ \gamma=\psi_{g} \circ \nu$, we have

$$
\left[\left(\pi_{\mathcal{I}_{B}} \circ \gamma\right)^{-1}(0)\right]=\left[\left(\psi_{g} \circ \nu\right)^{-1}(0)\right]=\sum_{\substack{s \in\left(\psi_{g} \circ \nu\right)^{-1}(0) \\ s \notin \nu^{-1}\left(p_{0}\right)}} u_{s} s+\sum_{s \in \nu^{-1}\left(p_{0}\right)} u_{s} s
$$

where $u_{s}$ are positive integers. Applying Lemma 2.8 to $\psi_{g}$ and $\psi_{g} \circ \nu$, it follows that $t=\sum_{s \in \nu^{-1}\left(p_{0}\right)} u_{s}$. By Remark[2.7 we have $\left[\pi_{\mathcal{I}_{B}}^{-1}(0)\right]=\sum_{M \subseteq \mathcal{F}_{C}^{\text {red }}}\left(\mu_{M} \mathcal{F}_{C}\right) M$. On the other hand, $W_{g}$ and $\mathcal{I}_{B}$ are isomorphic over $B \backslash\{0\}$, hence $\gamma: W_{g}^{\nu} \rightarrow \mathcal{I}_{B}$ 
is the normalization of $\mathcal{I}_{B}$. Applying again Lemma 2.8 to $\pi_{\mathcal{I}_{B}}$ and $\pi_{\mathcal{I}_{B}} \circ \gamma$, and using (8), it follows that

$$
\mu_{L} \mathcal{F}_{C}=\sum_{s \in \gamma^{-1}((0, L))} u_{s}=\sum_{s \in \nu^{-1}\left(p_{0}\right)} u_{s}=t .
$$

We obtain that $L \in \mathcal{F}_{C}$ and that condition (i) holds.

Suppose that $p_{0}$ is a node of $C$. Choose a smoothing $g: \mathcal{C} \rightarrow B$ of $C$ where $\mathcal{C}$ has a singularity of $A_{1}$-type at $p_{0}$. Let $\pi: \mathcal{X} \rightarrow \mathcal{C}$ be the blowup of $\mathcal{C}$ at $p_{0}$ and $f: \mathcal{X} \rightarrow B$ be the composed morphism $f:=g \circ \pi$. Write $f^{-1}(0)=Y \cup E$, where $Y$ is the normalization of $C$ at $p_{0}$ and $E$ is the exceptional component of $\pi$. Consider the $f$-Weierstrass scheme $W_{f} \hookrightarrow \mathcal{X}$ and let $\psi_{f}: W_{f} \rightarrow B$ be the associated finite morphism of degree 24. Since $W_{f}$ and $W_{g}$ are isomorphic over $B \backslash\{0\}$, it follows that $W_{g}^{\nu}$ is the normalization of $W_{f}$. Let $\bar{\nu}: W_{g}^{\nu} \rightarrow W_{f}$ be the normalization map. We get the following commutative diagram.

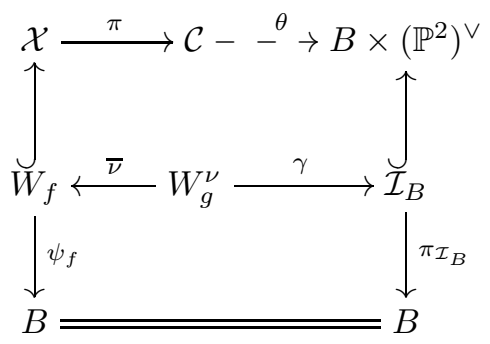

It follows from Lemma 2.2 that $\left[W_{f, 0}\right]=\sum_{i=1}^{18-\epsilon_{1}-\epsilon_{2}} q_{i}+\left(3+\epsilon_{1}\right) p_{1}+\left(3+\epsilon_{2}\right) p_{2}$, for some $\epsilon_{1}, \epsilon_{2} \in\{0,1\}$, where $\left\{p_{1}, p_{2}\right\}:=E \cap Y$ and $\left\{q_{1}, \ldots, q_{18-\epsilon_{1}-\epsilon_{2}}\right\} \subset Y-E$. The set $\gamma\left(\bar{\nu}^{-1}\left(p_{i}\right)\right)$ parametrizes the lines of $\mathcal{F}_{C}$ cutting $C$ with multiplicity at least 3 in $p_{0}$ and tangent to the branch of $p_{0}$ determined by $p_{i}$, for $i \in\{1,2\}$. In this way, we have $\gamma\left(\bar{\nu}^{-1}\left(p_{i}\right)\right)=\left(0, L_{i}\right)$, where $L_{i}$ is the unique line of the tangent cone of $C$ at $p_{0}$ which is tangent to the branch of $p_{0}$ determined by $p_{i}$, for $i \in\{1,2\}$. Arguing as in the case where $p_{0}$ is a cusp or a tacnode, we have that $\mu_{L_{i}} \mathcal{F}_{C}=3+\epsilon_{i}$, for $i \in\{1,2\}$. We obtain that $L_{i} \in \mathcal{F}_{C}$, for $i \in\{1,2\}$, and that condition (ii) holds.

We complete the proof for $C$ irreducible as follows. Suppose that $C$ is irreducible and that $L$ is an inflection line $L$ of type 0 of $C$. The non-reduced point $p_{0}$ of $L \cap C$ has multiplicity at most 4 , hence $p_{0}$ has multiplicity at most 2 in $\left[W_{g, 0}\right]$. Arguing again as in the case where $p_{0}$ is a cusp or a tacnode, it follows that $\mu_{L} \mathcal{F}_{C} \leq 2$. 
From now on, assume that $C$ is reducible. Suppose that $L$ is a line such that $L \subset C$. If $C$ is nodal and $\overline{C-L}$ is an irreducible smooth plane cubic, pick a regular smoothing $g: \mathcal{C} \rightarrow B$ of $C$ to general plane quartics, and consider the first diagram introduced in the proof. Let $s_{1}, \ldots, s_{6}$ be the points of Lemma 2.4. so that $\left\{s_{1}, \ldots, s_{6}\right\} \subset L \cap C^{s m} \cap W_{g}$. Recall that $\gamma\left(\nu^{-1}\left(s_{i}\right)\right)$ parametrizes the set of lines of $\mathcal{F}_{C}$ cutting $C$ with multiplicity at least 3 in $s_{i}$. Since any line different from $L$ cuts $C$ with multiplicity 1 in $s_{i}$, necessarily $\gamma\left(\nu^{-1}\left(s_{i}\right)\right)=(0, L)$, and hence, arguing again as in the case where $p_{0}$ is a cusp or a tacnode, we have $\mu_{L} \mathcal{F}_{C} \geq 6$. In the remaining cases, if we take a deformation of $C$ to nodal curves which are the union of $L$ and a smooth cubic, we see that $\mu_{L} \mathcal{F}_{C} \geq 6$, because $L$ is constant along this deformation.

Suppose that $L$ is a line of the tangent cone of $C$ at some $p_{0} \in C^{\operatorname{sing}}$. Then $L$ is the specialization of inflection lines of type 1 of a family of curves whose general member is irreducible with exactly one singular point of the same analytic type of $p_{0}$. Hence $\mu_{L} \mathcal{F}_{C} \geq t$, where $t \in\{3,4\}$, if $p_{0}$ is a node, $t=8$ if $p_{0}$ is a cusp, and $t=12$, if $p_{0}$ is a tacnode. To complete the proof, we only need to show that conditions (ii), (iii) and (iii) hold for a reducible curve. In what follows, we will use the above inequalities and that $\sum_{L \subseteq \mathcal{F}_{C}} \mu_{L} \mathcal{F}_{C}=24$.

Suppose that $C=Q_{1} \cup Q_{2}$, for smooth conics $Q_{1}, Q_{2}$. If $C$ is nodal and $L_{1}, \ldots, L_{8}$ are the lines of the tangent cone of $C$ at its nodes, then $\mu_{L_{i}} \mathcal{F}_{C} \geq 3$, and hence necessarily $\mu_{L_{i}} \mathcal{F}_{C}=3$, for $i \in\{1, \ldots, 8\}$. If $Q_{1}$ and $Q_{2}$ intersect each other transversally at 2 points and they are tangent at a point $r$, necessarily $\mu_{L_{0}} \mathcal{F}_{C}=12$ and $\mu_{L_{i}} \mathcal{F}_{C}=3$, for $i \in\{1, \ldots, 4\}$, where $L_{0}$ is the tangent cone of $C$ at the tacnode $r$ and $L_{1}, \ldots, L_{4}$ are the lines of the tangent cone of $C$ at its nodes.

Suppose that $C=Q \cup L_{1} \cup L_{2}$, for a smooth conic $Q$ and lines $L_{1}, L_{2}$. If $C$ is nodal, necessarily $\mu_{L_{1}} \mathcal{F}_{C}=\mu_{L_{2}} \mathcal{F}_{C}=6$ and $\mu_{L_{i}} \mathcal{F}_{C}=3$, for $i \in\{3, \ldots, 6\}$, where $L_{3}, \ldots, L_{6}$ are the tangents to $Q$ at the points of the set $Q \cap\left(L_{1} \cup L_{2}\right)$. If $Q$ is tangent to $L_{1}$ and transverse to $L_{2}$, necessarily $\mu_{L_{1}} \mathcal{F}_{C}=12, \mu_{L_{2}} \mathcal{F}_{C}=6$ and $\mu_{L_{3}} \mathcal{F}_{C}=\mu_{L_{4}} \mathcal{F}_{C}=3$, where $L_{3}, L_{4}$ are the tangents to $Q$ at the points of the set $Q \cap L_{2}$.

If $C=\bigcup_{i=1}^{4} L_{i}$, for lines $L_{1}, \ldots, L_{4}$, necessarily $\mu_{L_{i}} \mathcal{F}_{C}=6$, for $i \in\{1, \ldots, 4\}$.

Suppose that $C=Y \cup L$, for an irreducible plane cubic $Y$ and a line $L$. Set $k=3$ (respectively $k=1$ ) if $Y$ has a node (respectively a cusp). Assume that $Y \cap L$ are nodes and set $\left\{p_{1}, p_{2}, p_{3}\right\}:=Y \cap L$. Let $L_{i}$ be the tangent to $Y$ at $p_{i}$ for $i \in\{1,2,3\}$. We distinguish two cases. 
In the first case, $Y \cap L$ contains no inflectionary point of $Y$. If $Y$ is smooth, we can use Lemma 2.4. Thus, arguing as for the inflection lines of type 0 when $C$ is irreducible, it follows that there are 9 inflection lines of type 0 of $C$, which are exactly the inflection lines of $Y$, and necessarily $\mu_{L} \mathcal{F}_{C}=6, \mu_{L_{i}} \mathcal{F}_{C}=3$, for $i \in\{1,2,3\}$, and the 9 inflection lines of type 0 of $C$ have multiplicity 1 . If $Y$ is singular, pick a deformation of $C$ to plane curves which are the union of $L$ and a smooth plane cubic. It follows from Lemma 2.9 that $C$ has $k$ inflection lines of type 0 , and hence necessarily $\mu_{L} \mathcal{F}_{C}=6, \mu_{L_{i}} \mathcal{F}_{C}=3$, for $i \in\{1,2,3\}$, $\mu_{M} \mathcal{F}_{C}=3$ (respectively $\mu_{M} \mathcal{F}_{C}=8$ ), where $M$ is a line of the tangent cone at a node (respectively a cusp) of $Y$, and the $k$ inflection lines of type 0 of $C$ have multiplicity 1. We isolate the case in which $C$ is nodal in Remark 2.11.

In the second case, there is a positive integer $k^{\prime} \leq \min (k, 3)$ such that $p_{i}$ is an inflectionary point of $Y$, for $i \in\left\{1, \ldots, k^{\prime}\right\}$, where $k:=9$ if $Y$ is smooth. Pick a deformation of $C$ to plane curves which are the union of $Y$ and a general line intersecting $Y$ transversally. Then exactly two inflection lines specialize to $L_{i}$, for $i \in\left\{1, \ldots, k^{\prime}\right\}$, one of type 0 of multiplicity 1 and one of type 1 of multiplicity 3 , and $C$ has $k-k^{\prime}$ inflection lines of type 0 . Hence $\mu_{L} \mathcal{F}_{C}=6$, $\mu_{L_{i}} \mathcal{F}_{C}=4$ for $i \in\left\{1, \ldots, k^{\prime}\right\}, \mu_{L_{i}}=3$ for $i \in\left\{k^{\prime}+1, \ldots, 3\right\}, \mu_{M} \mathcal{F}_{C}=3$ (respectively $\mu_{M} \mathcal{F}_{C}=8$ ), where $M$ is a line of the tangent cone at a node (respectively a cusp) of $Y$, and the $k-k^{\prime}$ lines of type 0 of $C$ have multiplicity 1.

Suppose that $C=Y \cup L$, for an irreducible plane cubic $Y$ and a line $L$ such that $Y$ and $L$ are tangent at a point $t$. Notice that $t$ is a non-inflectionary point of $Y$, because $[C] \in \mathcal{V}$. Write $\{t, n\}:=Y \cap L$. Consider a deformation of $C$ to plane curves which are the union of $Y$ and a general line containing $n$ and intersecting $Y$ transversally. Then exactly 3 inflection lines of the general fiber of the deformation degenerate to $L$, of multiplicities respectively $6,3,3$, hence $\mu_{L} \mathcal{F}_{C}=12$, while the other inflection lines are constant along the family.

Notice that if $C$ is reducible, then the inflection lines of type 0 of $C$ have always multiplicity 1 . We get that conditions (ii), (iii) and (iii) hold.

Remark 2.11: For ease of reference we observe the following immediate consequence of Proposition 2.10 .

- If either $C$ is irreducible, with $C^{\text {sing }}$ consisting exactly of one tacnode, or if $C$ is the union of a line $L$ and a smooth cubic $Y$ such that $L$ and $Y$ are tangent at a non-inflectionary point of $Y$, then $\mathcal{F}_{C}$ contains at 
least 6 inflection lines of type 0 each one of which has multiplicity at most 2 .

- If $C$ is nodal and $C=Y \cup L$, where $Y$ is an irreducible cubic with a node and $L$ is a line such that $Y \cap L$ contains no inflectionary points of $Y$, then $\mathcal{F}_{C}$ consists of the line $L$ with multiplicity 6 , the three inflection lines at smooth points of $Y$ of type 0 with multiplicity 1 , and five lines of type 1 of multiplicity 3, two coming from the inflection lines through the node of $Y$, and three coming from inflection lines through the intersection $Y \cap L$ and different from $L$ (see also the proof of Proposition 2.10).

Proposition 2.12: Consider the natural action of $P G L(3)$ on $\operatorname{Sym}^{24}\left(\mathbb{P}^{2 \vee}\right)$. Let $[C] \in \mathcal{V}$ and $\delta, \gamma, \tau$ be respectively the number of nodes, cusps, tacnodes of $C$. Then the following conditions hold:

(i) if $\mathcal{F}_{C}$ is GIT-unstable, then either $\gamma \geq 2$ or $\tau \geq 1$;

(ii) if $\gamma=\tau=0$, then $\mathcal{F}_{C}$ is GIT-stable.

Proof. Throughout the proof, we assume that $\gamma \leq 1$ and $\tau=0$. For a point $p \in \mathbb{P}^{2}$, let $\mu_{p} \mathcal{F}_{C}:=\sum_{p \in L \subset \mathcal{F}_{C}} \mu_{L} \mathcal{F}_{C}$. By [MFK, Proposition 4.3] or [N] Theorem 4.17], $\mathcal{F}_{C}$ is GIT-semistable if and only if $\mu_{p} \mathcal{F}_{C} \leq 16$, for every $p \in \mathbb{P}^{2}$, and $\mu_{L} \mathcal{F}_{C} \leq 8$, for every $L \subset \mathcal{F}_{C}$, and GIT-stable if and only if both inequalities are strict. By Proposition 2.10, $\mu_{L} \mathcal{F}_{C} \leq 8$, and the equality holds if and only if $\gamma=1$. Thus, we only need to check that $\mu_{p} \mathcal{F}_{C} \leq 16$, with $\mu_{p} \mathcal{F}_{C}<16$ if $\gamma=0$, for every $p \in \mathbb{P}^{2}$.

So, fix $p \in \mathbb{P}^{2}$. Let $M_{0}^{p}$ be the set of inflection lines of type 0 of $C$ containing p. By Proposition 2.10 (iii), $\mu_{L} \mathcal{F}_{C} \leq 2$, for every $L \in M_{0}^{p}$. Let $M_{1}^{p}$ be the set of inflection lines of type 1 of $C$ containing $p$. We distinguish 3 cases.

(a) Suppose that $C$ is irreducible. If $p \in C^{\text {sing }}$, then $\mu_{p} \mathcal{F}_{C} \leq 8$, by Proposition 2.10. Hence we can assume $p \notin C^{\text {sing }}$. Let $\pi: C^{\nu} \rightarrow \mathbb{P}^{1}$ be the resolution of the projection map from $p$, where $C^{\nu}$ is the normalization of $C$, and $R$ be the ramification divisor of $\pi$.

First of all, assume that $p \notin C$. Then $\#\left\{q \in C^{\nu}: \nu_{q} R \geq 2\right\}=\#\left(M_{0}^{p} \cup M_{1}^{p}\right)$ and $\operatorname{deg} R=12-2 \delta-2 \gamma$, by Riemann-Hurwitz. Assume that $\gamma=0$ and, by contradiction, that $\mu_{p} \mathcal{F}_{C} \geq 16$. Observe that $\# M_{1}^{p} \leq 3$, otherwise $p$ would be a node, which is a contradiction. If $\# M_{1}^{p}=0$, then $\# M_{0}^{p} \geq 8$, hence $\operatorname{deg} R \geq 16$. If $\# M_{1}^{p}=1$, then $\# M_{0}^{p} \geq 6$, hence $\operatorname{deg} R \geq 14$. If $\# M_{1}^{p}=2$, then $\delta \geq 2$, otherwise $p$ would be a node, and $\# M_{0}^{p} \geq 4$ which implies $\operatorname{deg} R \geq 12$. If 
$\# M_{1}^{p}=3$, then $\delta \geq 3$, otherwise $p$ would be a node, and $\# M_{0}^{p} \geq 2$, implying $\operatorname{deg} R \geq 10$. In any case, we get a contradiction. Assume now that $\gamma=1$ and, by contradiction, that $\mu_{p} \mathcal{F}_{C}>16$. Let $L$ be the line of the tangent cone at the cusp of $C$, with $\mu_{L} \mathcal{F}_{C}=8$. If $p \notin L$, then $\mu_{p} \mathcal{F}_{C} \leq 16$, a contradiction. Hence $p \in L$ and $\# M_{1}^{p} \geq 1$. If $\# M_{1}^{p}=1$, then $\# M_{0}^{p} \geq 5$, hence $\operatorname{deg} R \geq 12$. If $\# M_{1}^{p}=2$, then $\delta \geq 1$ and $\# M_{0}^{p} \geq 3$, hence $\operatorname{deg} R \geq 10$. If $\# M_{1}^{p}=3$, then $\delta \geq 2$ and $\# M_{0}^{p} \geq 1$, hence $\operatorname{deg} R \geq 8$. In any case, we get a contradiction.

Suppose that $p \in C^{s m}$. Then $\nu_{p} R \leq 2$ and, if $T_{p} C$ is the tangent to $C$ at $p$, we have $\#\left\{q \in C^{\nu}: \nu_{q} R \geq 2\right\} \geq \#\left(\left(M_{0}^{p} \cup M_{1}^{p}\right)-\left\{T_{p} C\right\}\right)$. Notice that $\operatorname{deg} R=10-2 \delta-2 \gamma$, by Riemann-Hurwitz, and we can conclude arguing exactly as in the case $p \notin C$.

(b) Suppose that $C=Q_{1} \cup Q_{2}$, for conics $Q_{1}, Q_{2}$. Hence $C$ is nodal, because $\tau=0$. If $Q_{1}, Q_{2}$ are irreducible, then $p$ is contained in at most four inflection lines of $C$, and hence $\mu_{p} \mathcal{F}_{C} \leq 12$. If $Q_{1}$ is irreducible and $Q_{2}$ is the union of two lines $L_{1}, L_{2}$, then $\mu_{p} \mathcal{F}_{C} \leq 12$, where the equality holds if and only if either $p \in L_{1} \cap L_{2}$ or $p \in L_{i} \cap L \cap L^{\prime}$, for $i \in\{1,2\}$, where $L, L^{\prime}$ are components of $\mathcal{F}_{C}$ such that $\left\{L, L^{\prime}\right\} \cap\left\{L_{1}, L_{2}\right\}=\emptyset$. If $Q_{1}$ and $Q_{2}$ are reducible, i.e. if $C$ is the union of four lines $L_{1}, \ldots, L_{4}$, then $\mu_{p} \mathcal{F}_{C} \leq 12$, where the equality holds if and only if $p \in L_{i} \cap L_{j}$, for $i, j \in\{1, \ldots, 4\}$ and $i \neq j$.

(c) Suppose that $C=Y \cup L$, for an irreducible cubic $Y$ and a line $L$. By contradiction, assume $\mu_{p} \mathcal{F}_{C} \geq 16$. By Proposition 2.10, $\mu_{L} \mathcal{F}_{C}=6$ and $\mu_{L^{\prime}} \mathcal{F}_{C}=1$, for $L^{\prime} \in M_{0}^{p}$. We have $\# M_{1}^{p} \leq 2$ and, if $p \in C^{\text {sing }}$, then $\mu_{p} \mathcal{F}_{C} \leq 10$, a contradiction. If $p \in L-Y$ and $Y$ is smooth, then $\# M_{0}^{p} \geq 10$, a contradiction because $\# M_{0}^{p} \leq 9$, by Proposition 2.10. If $p \in L-Y$ and $Y$ has a node, then $\# M_{1}^{p} \leq 1$ and $\# M_{0}^{p} \geq 6$, a contradiction because $\# M_{0}^{p} \leq 3$, by Proposition 2.10. If $p \in L-Y$ and $Y$ has a cusp, then $\# M_{1}^{p} \leq 1$ and $\# M_{0}^{p} \geq 2$, a contradiction because $\# M_{0}^{p} \leq 1$, by Proposition 2.10 .

So we can assume $p \notin C^{\text {sing }} \cup L$. If $\mu_{L^{\prime}} \mathcal{F}_{C} \geq 3$, for some $L^{\prime} \subset \mathcal{F}_{C}$, then $p \in L^{\prime}$, otherwise $\mu_{p} \mathcal{F}_{C} \leq 15$, a contradiction. In this way, if $Y$ is smooth, then $\# M_{1}^{p}=3$ and hence also $\# M_{0}^{p} \geq 4$. We get a contradiction considering the projection $Y \rightarrow \mathbb{P}^{1}$ from $p$ and by Riemann-Hurwitz. If $Y$ has a node $q$, then $p$ is contained in the two lines of the tangent cone at $q$, hence $p=q \in C^{s i n g}$, a contradiction. If $Y$ has a cusp $q$ and $L_{0}$ is the line of the tangent cone at $q$, then $\# M_{1}^{p}=4$, with $L_{0} \in M_{1}^{p}$. We get a contradiction by considering the resolution $Y^{\nu} \rightarrow \mathbb{P}^{1}$ of the projection from $p$, where $Y^{\nu}$ is the normalization of $Y$, and by Riemann-Hurwitz. 


\section{Recovering quartics from inflection lines and inflection points}

Fix a line $L \subset \mathbb{P}^{2}$ and a point $p \in L$. Recall that $\mathcal{V} \subset \mathbb{P}^{14}$ is the variety parametrizing reduced plane quartic curves which are GIT-semistable with respect to the natural action of $P G L(3)$ and with finite stabilizers. Let $\mathcal{V}_{L} \subset \mathcal{V}$ be the locus defined as the closure in $\mathcal{V}$ of

$$
\mathcal{V}_{L}^{0}:=\left\{[C] \in \mathcal{V}: L \not \subset C \text { and } L \subset \mathcal{F}_{C}^{r e d}\right\} \text {. }
$$

Let $\mathcal{V}_{L, p} \subset \mathcal{V}_{L}$ be the locus defined as the closure in $\mathcal{V}_{L}$ of

$$
\mathcal{V}_{L, p}^{0}:=\left\{[C] \in \mathcal{V}_{L}^{0}: p \text { is the non-reduced point of } C \cap L\right\} .
$$

Lemma 3.1: Let $[C] \in \mathcal{V}$ with $C$ nodal and $L \subset \mathbb{P}^{2}$ be a line. Then:

(i) if $[C] \in \mathcal{V}_{L}^{0}$ and $C \cap L=\{p, q\} \subset C^{s m}$, where $p$ is the non-reduced point of $C \cap L$, and $p \neq q$, then

$$
T_{[C]} \mathcal{V}_{L} \simeq H^{0}\left(C, \mathcal{O}_{C}(4) \otimes \mathcal{O}_{C}(-2 p)\right)
$$

(ii) if $[C] \in \mathcal{V}_{L}^{0}$ and $C \cap L=\{p, q\}$, with $p$ a node of $C$ and $q \in C^{s m}$, and if $\nu: C^{\nu} \rightarrow C$ is the normalization of $C$ at $p$, with $\left\{p_{1}, p_{2}\right\}:=\nu^{-1}(p)$, where $L$ is the tangent to the branch of $p$ determined by $p_{1}$, then

$$
T_{[C]} \mathcal{V}_{L} \simeq H^{0}\left(C^{\nu}, \nu^{*} \mathcal{O}_{C}(4) \otimes \mathcal{O}_{C^{\nu}}\left(-2 p_{1}-p_{2}\right)\right)
$$

(iii) if $L \subset C$ and $p \in C^{s m} \cap L$, then $[C] \in \mathcal{V}_{L, p}$ and

$$
T_{[C]} \mathcal{V}_{L, p} \simeq H^{0}\left(C, \mathcal{O}_{C}(4) \otimes \mathcal{O}_{C}(-3 p)\right)
$$

Proof. Let $x, y, z$ be homogeneous coordinates of $\mathbb{P}^{2}$. Let $a_{0}, \ldots, a_{14}$ be the homogeneous coordinates of the projective space $\mathbb{P}^{14}$ parametrizing plane quartic curves. Let $\mathbb{C}[x, y, z]_{d}$ be the vector space of homogeneous polynomials of degree $d$ in $x, y, z$.

Suppose that $C$ is as in (ii) or (iii). First of all, we will show that $\mathcal{V}_{L}^{0}$ is smooth of dimension 12 at $[C]$. Up to an isomorphism of $\mathbb{P}^{2}$, we can assume that $L=Z(z)$. Notice that $[Z(F)] \in \mathcal{V}_{L}^{0}$ for some $F \in \mathbb{C}[x, y, z]_{4}$ if and only if $F(x, y, 0)$ is a binary quartic

$$
F(x, y, 0)=a_{0} x^{4}+a_{1} x^{3} y+a_{2} x^{2} y^{2}+a_{3} x y^{3}+a_{4} y^{4},
$$

where $\left[a_{0}, a_{1}, a_{2}, a_{3}, a_{4}\right] \in \mathbb{P}_{a_{0}, \ldots, a_{4}}^{4}$ and the binary quartic has at least a triple root. Then we have a rational map $\tau: \mathcal{V}_{L}^{0} \rightarrow L \times L$ defined as follows: if $\left[C^{\prime}\right] \in \mathcal{V}_{L}^{0}$ and $C^{\prime} \cap L=\left\{p^{\prime}, q^{\prime}\right\}$ with $p^{\prime} \neq q^{\prime}$ and $p^{\prime}$ is the non-reduced point 
of $C^{\prime} \cap L$, then we let $\tau\left(\left[C^{\prime}\right]\right)=\left(p^{\prime}, q^{\prime}\right)$. Near $[C]$, the map $\tau$ is defined by the assumption that $p$ and $q$ are different, its fibers are $\mathbb{A}_{a_{5}, \ldots, a_{14}}^{10}$, and hence $\mathcal{V}_{L}^{0}$ is smooth of dimension 12 at $[C]$. In particular, $\operatorname{dim}\left(T_{[C]} \mathcal{V}_{L}\right)=12$.

Now, suppose that $C$ is as in (iil). By Riemann-Roch,

$$
h^{0}\left(C, \mathcal{O}_{C}(4) \otimes \mathcal{O}_{C}(-2 p)\right)=12,
$$

hence to conclude the proof of (ii), we only need to show that the natural injective map $H^{0}\left(C, \mathcal{O}_{C}(4) \otimes \mathcal{O}_{C}(-2 p)\right) \hookrightarrow H^{0}\left(C, \mathcal{O}_{C}(4)\right)$ factors via an injective map

$$
H^{0}\left(C, \mathcal{O}_{C}(4) \otimes \mathcal{O}_{C}(-2 p)\right) \hookrightarrow T_{[C]} \mathcal{V}_{L}
$$

Indeed, assume that $C=Z(F)$, for $F \in \mathbb{C}[x, y, z]_{4}$, and, up to an isomorphism of $L$, that $F(x, y, 0)=x y^{3}$, i.e. $p=[1,0,0]$ and $q=[0,1,0]$.

Recall that a vector of $T_{[C]} \mathcal{V}$ is given by a first order deformation $F+\epsilon \cdot G$, where $G \in \mathbb{C}[x, y, z]_{4}$ and $\epsilon^{2}=0$. This vector belongs to $T_{[C]} \mathcal{V}_{L}$ if and only if $(F+\epsilon \cdot G)(x, y, 0)$ has at least a triple root. If we view $G$ as a section of $\mathcal{O}_{\mathbb{P}^{2}}(4)$ and we consider the surjective restriction map

$$
\rho: H^{0}\left(\mathbb{P}^{2}, \mathcal{O}_{\mathbb{P}^{2}}(4)\right) \rightarrow H^{0}\left(C, \mathcal{O}_{C}(4)\right),
$$

we obtain an identification $T_{[C]} \mathcal{V} \simeq H^{0}\left(C, \mathcal{O}_{C}(4)\right)$. Notice that the vector space $H^{0}\left(C, \mathcal{O}_{C}(4) \otimes \mathcal{O}_{C}(-2 p)\right)$ is the image via $\rho$ of the set of sections of $\mathcal{O}_{\mathbb{P}^{2}}(4)$ induced by polynomials $\bar{G} \in \mathbb{C}[x, y, z]_{4}$ such that $Z(\bar{G})$ and $C$, and hence $Z(\bar{G})$ and $L$, intersect each other with multiplicity at least 2 in $p$, i.e. polynomials $\bar{G}$ such that

$$
\bar{G}(x, y, 0)=y^{2} H, \quad \text { for some } H \in \mathbb{C}[x, y]_{2} .
$$

In this case, write $H=3 H^{\prime} x+a y^{2}$, where $H^{\prime} \in \mathbb{C}[x, y]_{1}$ and $a \in \mathbb{C}$. Then

$$
\begin{aligned}
(F+\epsilon \cdot \bar{G})(x, y, 0) & =F(x, y, 0)+\epsilon \cdot \bar{G}(x, y, 0)=x y^{3}+\epsilon \cdot y^{2} H \\
& =x y^{3}+\epsilon \cdot\left(a y^{4}+3 H^{\prime} x y^{2}\right)=\left(y+\epsilon \cdot H^{\prime}\right)^{3}(x+\epsilon \cdot a y),
\end{aligned}
$$

where in the last equation we used the relation $\epsilon^{2}=0$. We see that

$$
(F+\epsilon \cdot \bar{G})(x, y, 0)
$$

has at least a triple root, hence it defines a vector of $T_{[C]} \mathcal{V}_{L}$, and hence there exists an injective map as required in (11). 
Suppose now that $C$ is as in (iii). Set $\mathcal{L}:=\nu^{*} \mathcal{O}_{C}(4) \otimes \mathcal{O}_{C^{\nu}}\left(-2 p_{1}-p_{2}\right)$. Since $g_{C^{\nu}}=2$, we have $h^{0}\left(C^{\nu}, \mathcal{L}\right)=12$, by Riemann-Roch. As before, to conclude the proof of (iii) it is enough that there is an injective map $H^{0}\left(C^{\nu}, \mathcal{L}\right) \hookrightarrow T_{[C]} \mathcal{V}_{L}$.

Indeed, let $W \subset H^{0}\left(C, \mathcal{O}_{C}(4)\right)$ be the subspace obtained as the image via the restriction map $\rho: H^{0}\left(\mathbb{P}^{2}, \mathcal{O}_{\mathbb{P}^{2}}(4)\right) \rightarrow H^{0}\left(C, \mathcal{O}_{C}(4)\right)$ of the set of sections of $\mathcal{O}_{\mathbb{P}^{2}}(4)$ induced by polynomials $\bar{G}$ satisfying (12). Arguing as for (1), we have an injective map $W \hookrightarrow T_{[C]} \mathcal{V}_{L}$. If $W^{\prime} \subset H^{0}\left(C, \mathcal{O}_{C}(4)\right)$ is the subspace of sections vanishing on $p$, then $W \subset W^{\prime}$. Let $\nu^{*}: H^{0}\left(C, \mathcal{O}_{C}(4)\right) \rightarrow H^{0}\left(C^{\nu}, \nu^{*} \mathcal{O}_{C}(4)\right)$ be the injective map induced by pullback of sections. Since $p$ is a node, any section of $H^{0}\left(C^{\nu}, \nu^{*} \mathcal{O}_{C}(4) \otimes \mathcal{O}_{C^{\nu}}\left(-p_{1}-p_{2}\right)\right)$ descends to a section of $\mathcal{O}_{C}(4)$ vanishing on $p$. Thus the restricted map $\left.\nu^{*}\right|_{W^{\prime}}: W^{\prime} \rightarrow H^{0}\left(C^{\nu}, \nu^{*} \mathcal{O}_{C}(4) \otimes \mathcal{O}_{C^{\nu}}\left(-p_{1}-p_{2}\right)\right)$ is an isomorphism, identifying $W$ with $H^{0}\left(C^{\nu}, \mathcal{L}\right)$, by the definition of $W$. Hence there is an injective map $H^{0}\left(C^{\nu}, \mathcal{L}\right) \hookrightarrow T_{[C]} \mathcal{V}_{L}$, as required.

To conclude the proof, suppose that $C$ is as in (iii), with $C=Z(F), F \in$ $\mathbb{C}[x, y, z]_{4}$. Recall that $L=Z(z)$ and $p=[1,0,0]$, hence $F(x, y, 0)=0$. Let $Z(H)$ be a plane quartic curve such that $L \not \subset Z(H)$ and $[Z(H)] \in \mathcal{V}_{L, p}$. Define

$$
\mathcal{C}:=\left\{(q, t) \in \mathbb{P}^{2} \times \mathbb{A}_{t}^{1} \text { such that } F_{t}(q):=t \cdot H(q)+F(q)=0\right\} \subseteq \mathbb{P}^{2} \times \mathbb{A}_{t}^{1} .
$$

If $f: \mathcal{C} \rightarrow \mathbb{A}_{t}^{1}$ is the restriction of the second projection morphism, then $f$ is a deformation of $C$ to curves of $\mathcal{V}_{L, p}$, because $F_{t}(x, y, 0)=t \cdot H(x, y, 0)$, and hence $[C] \in \mathcal{V}_{L, p}$.

The vector space $H^{0}\left(C, \mathcal{O}_{C}(4) \otimes \mathcal{O}_{C}(-3 p)\right)$ is the image via the restriction map $\rho: H^{0}\left(\mathcal{O}_{\mathbb{P}^{2}}(4)\right) \rightarrow H^{0}\left(\mathcal{O}_{C}(4)\right)$ of the set of sections of $\mathcal{O}_{\mathbb{P}^{2}}(4)$ induced by polynomials $G \in \mathbb{C}[x, y, z]_{4}$ such that $Z(G)$ and $L$ intersect each other with multiplicity at least 3 in $p$, i.e. satisfying

$$
G(x, y, 0)=y^{3} H, \quad \text { for some } H \in \mathbb{C}[x, y]_{1} .
$$

On the other hand, a first order deformation $F+\epsilon \cdot G$ of $C$ is a vector of $T_{[C]} \mathcal{V}_{L, p}$, if $(F+\epsilon \cdot G)(x, y, 0)$ has at least a triple root in $p$. Then, since $(F+\epsilon \cdot G)(x, y, 0)=\epsilon G(x, y, 0)$, the vector space $T_{[C]} \mathcal{V}_{L, p}$ is given by the first order deformations $F+\epsilon \cdot G$, for $G$ satisfying (13). In this way, $T_{[C]} \mathcal{V}_{L, p} \simeq$ $H^{0}\left(C, \mathcal{O}_{C}(4) \otimes \mathcal{O}_{C}(-3 p)\right)$.

LEMma 3.2: Let $\left[C_{1}\right] \in \mathcal{V}$, where $C_{1}$ is a nodal plane quartic curve which is the union of an irreducible cubic $Y$ with a node and a line $L$. Assume that $Y \cap L$ contains no inflectionary points of $Y$. If $\mathcal{F}_{C_{1}}=\mathcal{F}_{C_{2}}$, for some $\left[C_{2}\right] \in \mathcal{V}$, then 
$C_{1}=C_{2}$. In particular, the morphism $\mathcal{F}: \mathcal{V} \rightarrow \operatorname{Sym}^{24}\left(\mathbb{P}^{2} \vee\right)$ of Proposition 2.5 is generically finite onto its image.

Proof. Set $\mathcal{F}:=\mathcal{F}_{C_{1}}=\mathcal{F}_{C_{2}}$. First of all, we will show that $C_{2}$ is a nodal plane quartic which is the union of an irreducible cubic with a node and a line. Indeed, by Remark 2.11, $\mathcal{F}$ consists of three lines of type 0 of multiplicity 1 , five lines of type 1 of multiplicity 3 and one degenerate line of multiplicity 6 . By Proposition 2.10 (ii), $C_{2}$ does not contain a cusp or a tacnode, i.e. $C_{2}$ is nodal. Again by Proposition 2.10 (1), $C_{2}$ is reducible, otherwise $\mathcal{F}$ would contain an even number of lines of multiplicity 3 or 4 . By Proposition 2.10 (iii), $C_{2}$ contains exactly one linear component, hence $C_{2}$ is a nodal plane quartic which is the union of an irreducible cubic $W$ and a line $M$, and $W$ has a node, otherwise $\mathcal{F}$ would contain at most three lines of multiplicity 3 .

The linear component both of $C_{1}$ and of $C_{2}$ is the reduction of the unique component of multiplicity 6 of $\mathcal{F}$, hence $L=M$. By contradiction, assume that $Y \neq W$. Let $Y^{\vee} \subset\left(\mathbb{P}^{2}\right)^{\vee}$ and $W^{\vee} \subset\left(\mathbb{P}^{2}\right)^{\vee}$ be the dual curves of $Y$ and $W$. Then $Y^{\vee} \neq W^{\vee}$. Since $Y$ and $W$ are irreducible cubics with a node, $Y^{\vee}$ and $W^{\vee}$ have degree 4 . But $Y^{\vee}$ and $W^{\vee}$ intersect each other at five smooth points, corresponding to the five components of multiplicity 3 of $\mathcal{F}$, and at three cusps, corresponding to the three components of multiplicity 1 of $\mathcal{F}$, contradicting Bézout's theorem.

It follows from the first part of the proof that the cardinality of the fiber of $\mathcal{F}$ over $\mathcal{F}\left(\left[C_{1}\right]\right)$ is one; then by semicontinuity $\mathcal{F}$ is generically finite onto its image.

For every line $L \subset \mathbb{P}^{2}$ and for every point $p \in L$, consider the variety $\mathcal{V}_{L, p}$ defined in (10). Notice that $\mathcal{V}_{L, p}$ is irreducible, because it is the intersection of $\mathcal{V}$ with a linear subspace of $\mathbb{P}^{14}$ of codimension 3. In the proof of the next Theorem, we will follow the same strategy of [CS1, Theorem 5.2.1].

Theorem 3.3: Consider a line $L \subset \mathbb{P}^{2}$ and a point $p \in L$. Consider the morphism $\mathcal{F}: \mathcal{V} \rightarrow \operatorname{Sym}^{24}\left(\mathbb{P}^{2 \vee}\right)$ of Proposition 2.5. Then $\left.\mathcal{F}\right|_{\mathcal{V}_{L, p}}$ is injective on a non-empty open subset of $\mathcal{V}_{L, p}$.

Proof. Let $C_{0}$ be a nodal plane quartic curve which is the union of an irreducible cubic $Y$ with a node and $L$, with $p \in C_{0}^{s m} \cap L$, and such that $Y \cap L$ contains no inflectionary points of $Y$. Then $\left[C_{0}\right] \in \mathcal{V}_{L, p}$, by Lemma 3.1 (iii). Recall the configuration $\mathcal{F}_{C_{0}}$ in Remark2.11. Set $\left\{p_{1}, p_{2}, p_{3}\right\}:=Y \cap L$ and let $L_{i} \subset \mathcal{F}_{C_{0}}^{\text {red }}$ be 
the tangent to $Y$ at $p_{i}$, for $i \in\{1,2,3\}$. Let $p_{4}$ be the node of $Y$ and $L_{4} \subset \mathcal{F}_{C_{0}}^{r e d}$ be one of the two lines of the tangent cone of $C_{0}$ at $p_{4}$. Let $L_{5}, L_{6}, L_{7}$ be the inflection lines of type 0 of $C_{0}$ and $p_{i}$ be the non-reduced point of $L_{i} \cap C_{0}$, for $i \in\{5,6,7\}$.

Let $\mathcal{C} \rightarrow B$ be a smoothing of $C_{0}$ to general curves of $\mathcal{V}_{L, p}$. By contradiction, assume the existence of a one parameter family of curves $\mathcal{W} \rightarrow B$ such that $C_{b} \neq W_{b} \in \mathcal{V}_{L, p}$, for $b \in B \backslash\{0\}$, and $\mathcal{F}_{C_{b}}=\mathcal{F}_{W_{b}}$, for $b \in B$. If $b \in B \backslash\{0\}$, then Proposition 2.10 (iii) implies that the maximum multiplicity of a component of $\mathcal{F}_{C_{b}}$ is 2 , and hence that $W_{b}$ is smooth, because $\mathcal{F}_{C_{b}}=\mathcal{F}_{W_{b}}$. We have two cases.

CASE 1. Assume that $\left[W_{0}\right] \in \mathcal{V}$. Since $\mathcal{F}_{C_{0}}=\mathcal{F}_{W_{0}}$, by Lemma 3.2 we have $C_{0}=W_{0}$. We will show that $\left.\mathcal{F}\right|_{\mathcal{V}_{L, p}}$ is an immersion at $C_{0}$, implying that $C_{b}=$ $W_{b}$, for $b \in B \backslash\{0\}$, which is a contradiction. Let $\nu: C_{0}^{\nu} \rightarrow C_{0}$ and $\nu_{Y}: Y^{\nu} \rightarrow Y$ be respectively the normalizations of $C_{0}$ and $Y$, where $C_{0}^{\nu}=Y^{\nu} \cup L$. Set $\left\{p_{i, 1}, p_{i, 2}\right\}:=\nu^{-1}\left(p_{i}\right)$, for $i \in\{1,2,3,4\}$, where $p_{i, 1} \in Y^{\nu}$, for $i \in\{1,2,3\}$, and let $\widehat{\mathcal{V}}$ be the locus of $\mathcal{V}_{L, p}$ parametrizing curves with $L_{1}, \ldots, L_{7}$ as inflection lines. Then $\left[C_{0}\right] \in \widehat{\mathcal{V}}$ and, up to switching $p_{4,1}$ and $p_{4,2}$, by Lemma 3.1 we have

$$
T_{\left[C_{0}\right]} \widehat{\mathcal{V}} \subseteq H^{0}\left(Y^{\nu}, \mathcal{L}_{1}\right) \oplus H^{0}\left(L, \mathcal{L}_{2}\right)
$$

where

$$
\mathcal{L}_{1}:=\nu_{Y}^{*} \mathcal{O}_{Y}(4) \otimes \mathcal{O}_{Y^{\nu}}\left(-2\left(\sum_{i=1}^{4} p_{i, 1}\right)-p_{4,2}-2 p_{5}-2 p_{6}-2 p_{7}\right) \simeq \mathcal{O}_{\mathbb{P}^{1}}(-3)
$$

and

$$
\mathcal{L}_{2}:=\mathcal{O}_{L}(4) \otimes \mathcal{O}_{L}\left(-p_{1,2}-p_{2,2}-p_{3,2}-3 p\right) \simeq \mathcal{O}_{\mathbb{P}^{1}}(-2)
$$

In this way, $T_{\left[C_{0}\right]} \widehat{\mathcal{V}} \subseteq H^{0}\left(\mathbb{P}^{1}, \mathcal{O}_{\mathbb{P}^{1}}(-3)\right) \oplus H^{0}\left(\mathbb{P}^{1}, \mathcal{O}_{\mathbb{P}^{1}}(-2)\right)=0$. Denoting by $F$ the fiber over the image of $\left[C_{0}\right]$ under the morphism $\left.\mathcal{F}\right|_{\mathcal{V}_{L, p}}$, that is

$$
F:=\left(\left.\mathcal{F}\right|_{\mathcal{V}_{L, p}}\right)^{-1}\left(\left.\mathcal{F}\right|_{\mathcal{V}_{L, p}}\left(\left[C_{0}\right]\right)\right)
$$

we have the inclusion of tangent spaces

$$
T_{\left[C_{0}\right]} F \subseteq T_{\left[C_{0}\right]} \widehat{\mathcal{V}}=0
$$

hence $\left.\mathcal{F}\right|_{\mathcal{V}_{L, p}}$ is an immersion at $\left[C_{0}\right]$.

CAsE 2. Assume that $\left[W_{0}\right] \notin \mathcal{V}$. If $W_{0}$ is GIT-semistable, then either it has an infinite stabilizer, or it is non-reduced, and hence it is a double conic. In any case, either $W_{0}$ is GIT-unstable, or it has an infinite stabilizer. By the 
GIT-semistable replacement property (see [CS1, Section 2.1]), up to a finite base change totally ramified over $0 \in B$, we can assume that there are a family $\mathcal{Z} \rightarrow B$ of curves of $\mathcal{V}$ and a morphism $\rho: B \backslash\{0\} \rightarrow P G L(3)$ such that $Z_{b}=$ $W_{b}^{\rho(b)}$ for every $b \in B \backslash\{0\}$. In particular, $\mathcal{F}_{Z_{b}}=\left(\mathcal{F}_{W_{b}}\right)^{\rho(b)}$, for $b \in B \backslash\{0\}$. Recall that $\mathcal{F}_{C_{0}}=\mathcal{F}_{W_{0}}$, hence $\mathcal{F}_{C_{0}}$ and $\mathcal{F}_{Z_{0}}$ are limits of $P G L(3)$-conjugate families of configuration of lines of $\operatorname{Sym}^{24}\left(\mathbb{P}^{2 \vee}\right)$. In this way, if $O r b_{\mathcal{F}_{C_{0}}}$ is the $P G L(3)$-orbit of $\mathcal{F}_{C_{0}}$, and $\overline{O r b}_{\mathcal{F}_{C_{0}}}$ is its closure in $\operatorname{Sym}^{24}\left(\mathbb{P}^{2 \vee}\right)$, then $\mathcal{F}_{Z_{0}} \in$ $\overline{\operatorname{Orb}}_{\mathcal{F}_{C_{0}}}$.

If $\mathcal{F}_{Z_{0}} \in O r b_{\mathcal{F}_{C_{0}}}$, then $\mathcal{F}_{Z_{0}}=\left(\mathcal{F}_{C_{0}}\right)^{g}=\mathcal{F}_{C_{0}^{g}}$, for some $g \in P G L(3)$, and hence $Z_{0}=C_{0}^{g}$, by Lemma 3.2. In this way, we can assume that a family which is a semistable replacement of $\mathcal{W} \rightarrow B$ has $C_{0}$ as central fiber and, after acting with $P G L(3)$, we can argue as in Case 1, obtaining a contradiction.

If $\mathcal{F}_{Z_{0}} \in \overline{O r b}_{\mathcal{F}_{C_{0}}}-O r b_{\mathcal{F}_{C_{0}}}$, then $\mathcal{F}_{Z_{0}}$ is GIT-unstable, because $\mathcal{F}_{C_{0}}$ is GITstable, by Proposition 2.12 (iii). Thus, $Z_{0}$ contains either two cusps or one tacnode, by Proposition 2.12 (ii). Notice that $\mathcal{F}_{Z_{0}}$ is the degeneration of configurations of lines conjugate to $\mathcal{F}_{C_{0}}$.

If $Z_{0}$ contains two cusps, then by Proposition 2.10 (ii) there are two distinct components $M_{1}, M_{2} \subset \mathcal{F}_{Z_{0}}$ such that $\mu_{M_{1}} \mathcal{F}_{Z_{0}}=\mu_{M_{2}} \mathcal{F}_{Z_{0}}=8$. Then both $M_{1}$ and $M_{2}$ are degenerations of a set of inflection lines whose multiplicities sum up to 8 . This is not possible because, by Remark 2.11, $\mathcal{F}_{C_{0}}$ consists of three lines of multiplicity 1 , five lines of multiplicity 3 and one line of multiplicity 6 .

If $Z_{0}$ contains one tacnode, then arguing as in CS1, Proposition 5.1.1, page 241 , we can assume that $Z_{0}$ is either irreducible with $Z_{0}^{\text {sing }}$ consisting exactly of one tacnode, or $Z_{0}$ is the union of a line and a smooth irreducible cubic that are tangent at a non-inflectionary point of the cubic. By Remark 2.11, $Z_{0}$ has at least six inflection lines of type 0 each one of which has multiplicity at most 2. This is not possible, because $\mathcal{F}_{C_{0}}$ contains only three lines of multiplicity at most two.

\section{Inflection lines of plane cubics}

Let $C \subset \mathbb{P}^{2}$ be a smooth plane cubic over a field $k$ and let $T_{C} \subset\left(\mathbb{P}^{2}\right)^{\vee}$ be the locus of inflection lines to the cubic $C$. The purpose of this section is to show that the cubic $C$ can be reconstructed from the subset $T_{C} \subset\left(\mathbb{P}^{2}\right)^{\vee}$ if the characteristic of $k$ is different from three (Theorem 4.6). Note that we need not assume that the field $k$ is algebraically closed. 
Inflection points to a smooth plane cubic correspond to cubic roots of the class of a line and are therefore a torsor under the three-torsion subgroup of the Jacobian of the curve. Thus, in the case of fields of characteristic three, there is little hope of reconstructing a smooth plane cubic from its inflection lines. More specifically, let $k$ be a field of characteristic three; for $\lambda \in k^{\times}$let $C_{\lambda}$ be the smooth projective cubic

$$
C_{\lambda}: x^{3}+y^{3}+z^{3}-\lambda x y z=0 .
$$

Let $F_{\lambda}$ be the set of points $\{[1,-1,0],[1,0,-1],[0,1,-1]\}$ of $C_{\lambda}$. It is easy to check that $F_{\lambda}$ is the set of inflection points of the curve $C_{\lambda}$ and that the inflection lines to $C_{\lambda}$ are the lines $\{x=0, y=0, z=0\}$. We conclude that the inflection points and the inflection lines for all the cubics $C_{\lambda}$ coincide, so that in this case it is impossible to reconstruct a smooth plane cubic from the set of inflection lines, even if all the inflection points are given. For this reason, we assume that the characteristic of the ground field is not three.

The reconstruction method that we follow proceeds essentially by identifying the dual curve to the cubic $C$. If the characteristic of $k$ is different from two, we show that there is a unique reduced sextic in $\left(\mathbb{P}^{2}\right)^{\vee}$ having cusps at the nine points in $T_{C}$. In the characteristic two case, there are two possibilities. If the $j$-invariant of the curve $C$ is non-zero, then there is a unique plane cubic in $\left(\mathbb{P}^{2}\right)^{\vee}$ containing $T_{C}$ and this cubic is isomorphic to $C$. If the $j$-invariant of the curve $C$ is zero, then there is a pencil of plane cubics containing $T_{C}$, but there is a unique cubic in the pencil with vanishing $j$-invariant; this curve is isomorphic to $C$.

To solve the reconstruction problem we study linear systems associated to the anticanonical divisor of the blow up $S_{C}$ of $\left(\mathbb{P}^{2}\right)^{\vee}$ along $T_{C}$. With the unique exception $(\operatorname{char}(k), j(C))=(2,0)$, we show that the anticanonical class on $S_{C}$ is linearly equivalent to a unique effective divisor and that twice the anticanonical linear system is a base-point free pencil (Corollary 4.4). This implies that the points of $T_{C}$ are the base locus of a Halphen pencil of index two. In the exceptional case the anticanonical linear system itself is a base point free pencil.

We start by proving a lemma that allows us to compute dimensions of certain special kinds of linear systems on surfaces.

Lemma 4.1: Let $X$ be a smooth projective surface over a field and let $N$ be an effective nef divisor on $X$ such that the equality $N^{2}=0$ holds. If the divisor 
$N$ is connected and reduced, then the linear system $|N|$ has dimension at most one, and it is base-point free if it has dimension one. If the linear system $|N|$ is base-point free, then no element of $|2 N|$ is connected and reduced.

Proof. Assume that $N$ is connected and reduced; we first show that the linear system $|N|$ either has dimension zero or it is base-point free. Write $N=M+F$, where $F$ is the fixed divisor of $|N|$ and $M$ is a base-component free divisor. By the assumptions, the equalities

$$
0=N^{2}=(M+F)^{2}=M \cdot(M+F)+F(M+F)
$$

hold; since $M+F$ is nef and $M$ and $F$ are both effective, we deduce that the last two summands in the previous equality are non-negative so that also the equalities $M \cdot(M+F)=F \cdot(M+F)=0$ hold. Similarly, the divisor $M$ is nef, because it has no base components, and hence $M \cdot M=M \cdot F=0$. Since the divisor $N$ is reduced, the divisors $M$ and $F$ have no components in common, and since $N$ is connected, the equality $M \cdot F=0$ implies that either $M$ or $F$ vanishes. If $M=0$, then the linear system $|N|$ has dimension zero and we are done. If $N=0$, then the linear system $|N|$ is base-component free, and the number of base points is bounded above by $N^{2}=0$, so that $|N|$ is base-point free.

Thus we reduce to the case in which $N$ is non-zero and the linear system $|N|$ is base-point free, and we need to show that the dimension of $|N|$ is one. Let $\varphi: X \rightarrow|N|^{\vee}$ be the morphism determined by $N$. Since $N^{2}=0$, the image $R$ of $\varphi$ is a curve. The divisor $N$ is the inverse image of a hyperplane $H$ in $|N|^{\vee}$ under the morphism $\varphi$, and since by assumption $N$ is connected and reduced, we deduce that the intersection of the hyperplane $H$ with $R$ is also reduced and connected. Since $R$ is a curve, it follows that $R$ has degree one and it is therefore a line. Because $R \subset|N|^{\vee}$ is not contained in any hyperplane, it follows that $R=|N|^{\vee}$, and we conclude that the dimension of $|N|$ is one, as required.

To prove the last assertion, note that the inequality $\operatorname{dim}|N| \geq 1$ implies the inequality $\operatorname{dim}|2 N| \geq 2$, so that the linear system $|2 N|$ cannot contain a connected and reduced divisor by the first part of the lemma.

The following result shows that the set of inflection lines to a smooth plane cubic forms the base point of a Halphen pencil of index two with a unique exception; we analyze separately the exception. We chose a quick explicit argument to handle the case of characteristic two. From a characteristic-free point 
of view, the case of vanishing $j$-invariant is special because it is the only case in which three inflection lines to a smooth plane cubic are concurrent. This fact, combined with the inseparability of the Gauss map in characteristic two, accounts for the exception.

Lemma 4.2: Let $k$ be a field of characteristic different from three. Let $C \subset \mathbb{P}^{2}$ be a smooth cubic curve over $k$, and let $S_{C}$ be the blow up of the projective plane $\left(\mathbb{P}^{2}\right)^{\vee}$ dual to $\mathbb{P}^{2}$ at the points corresponding to the inflection lines to $C$. The anticanonical divisor $-K_{S_{C}}$ of $S_{C}$ is nef and linearly equivalent to an effective divisor; the linear system $\left|-2 K_{S_{C}}\right|$ is base-point free. Moreover, extending the ground field if necessary, the linear system $\left|-2 K_{S_{C}}\right|$ contains a connected and reduced divisor, unless $\operatorname{char}(k)=2$ and the $j$-invariant of $C$ vanishes.

Proof. Let $T_{C} \subset\left(\mathbb{P}^{2}\right)^{\vee}$ denote the set of inflection lines to the cubic $C$, and recall that $T_{C}$ consists of nine distinct points. The surface $S_{C}$ is the blow up of the projective plane $\left(\mathbb{P}^{2}\right)^{\vee}$ at the nine points in $T_{C}$. Since there is always a plane cubic curve containing any nine points, we deduce that the anticanonical divisor of $S_{C}$ is linearly equivalent to an effective divisor, and that the linear system $\left|-2 K_{S_{C}}\right|$ contains non-reduced divisors corresponding to twice the divisors in $\left|-K_{S_{C}}\right|$.

Let $C^{\vee}$ denote the strict transform in $S_{C}$ of the curve dual to $C$. The curve $C^{\vee}$ is an irreducible element of the linear system $-2 K_{S}$ on $S_{C}$ and it is reduced if and only if $\operatorname{char}(k) \neq 2$. Since the equality $\left(C^{\vee}\right)^{2}=0$ holds, the anticanonical divisor $-K_{S_{C}}$ is nef. To conclude we show that the linear system $\left|-2 K_{S_{C}}\right|$ contains a connected and reduced divisor, possibly after an extension of the ground field; the result then follows applying Lemma 4.1

If the characteristic of the field $k$ is different from two, then the linear system $\left|-2 K_{S_{C}}\right|$ contains the integral divisor $C^{\vee}$ and we are done.

Suppose that the $j$-invariant of $C$ is non-zero. We show, assuming that the ground field is algebraically closed, that there is a connected reduced divisor in the linear system $\left|-2 K_{S_{C}}\right|$ not containing the reduction $\left(C^{\vee}\right)_{\text {red }}$ as a component. Let

$$
\mathcal{H}:\left\{\lambda_{0}\left(x^{3}+y^{3}+z^{3}\right)-3 \lambda_{1} x y z=0\right\}
$$

be the pencil of plane cubics parameterized by $\left[\lambda_{0}, \lambda_{1}\right] \in \mathbb{P}^{1}$; the pencil $\mathcal{H}$ is also known as the Hesse pencil. Over an algebraically closed field of characteristic different from three, any isomorphism between plane cubics is induced, up to 
composition with a translation, by an isomorphism sending an inflection point to an inflection point, and is therefore realized by a projective equivalence. Thus, over an algebraically closed field of characteristic different from three, every non-singular plane cubic is projectively equivalent to a curve appearing in the Hesse pencil, since the $j$-invariant in the family $\mathcal{H}$ is non-constant. To prove the result we shall assume that the curve $C$ is the curve

$$
C_{\lambda}:\left\{x^{3}+y^{3}+z^{3}-3 \lambda x y z=0\right\}
$$

in the pencil $\mathcal{H}$ corresponding to the parameter $[1, \lambda] \in \mathbb{P}^{1}$. The inflection points of the curve $C_{\lambda}$ are the nine points

$$
[1, \epsilon, 0], \quad[1,0, \epsilon], \quad[0,1, \epsilon], \quad \text { where } \epsilon^{3}+1=0,
$$

and the corresponding inflection lines are the lines with equations $x+\omega y+\lambda \omega^{2} z=0, \quad x+\lambda \omega^{2} y+\omega z=0, \quad \lambda \omega^{2} x+y+\omega z=0, \quad$ where $\omega^{3}=1$. It is therefore clear that the sextic $\left(\lambda x^{2}-y z\right)\left(\lambda y^{2}-x z\right)\left(\lambda z^{2}-x y\right)$ corresponds to an element $\Sigma_{\lambda}$ of $\left|-2 K_{S_{C}}\right|$ that is connected and reduced, if $\lambda \neq 0$. Since the divisor $\Sigma_{\lambda}$ is a union of conics, it follows that $\Sigma_{\lambda}$ does not contain $C^{\vee}$. To conclude, observe that the $j$-invariant of the curve $C_{0}$ vanishes.

Remark 4.3: The exception mentioned in Lemma 4.2 is necessary. Let $F \subset \mathbb{P}^{2}$ be the Fermat cubic curve with equation $x^{3}+y^{3}+z^{3}=0$ over a field of characteristic different from three. As in the proof of Lemma 4.2, the inflection points of the curve $F$ are the points with coordinates

$$
[1, \epsilon, 0], \quad[1,0, \epsilon], \quad[0,1, \epsilon], \quad \text { where } \epsilon^{3}+1=0,
$$

and the corresponding inflection lines are the points with coordinates $\left[1,-\epsilon^{2}, 0\right]$ in $\left(\mathbb{P}^{2}\right)^{\vee}$ (up to permutations). Therefore, the coordinates of the inflection points of the Fermat cubic involve cubic roots of -1 , while the coordinates of its inflection lines involve cubic roots of 1: in the case of a field of characteristic two, the configuration of inflection points and the configuration of inflection lines are projectively equivalent! Thus we see that the set $T$ of the points in (15) is the base locus of the Hesse pencil $\mathcal{H}$, and we conclude that the anticanonical linear system of the surface $S_{F}$ obtained by blowing up $\mathbb{P}^{2}$ along $T$ has dimension at least one. It follows at once that the dimension of the anticanonical linear system on $S_{F}$ is one. 
COROllary 4.4: Let $k$ be a field of characteristic different from three. Let $C \subset \mathbb{P}^{2}$ be a smooth cubic curve over $k$, and let $S_{C}$ be the blow up of the projective plane $\left(\mathbb{P}^{2}\right)^{\vee}$ dual to $\mathbb{P}^{2}$ at the points corresponding to the inflection lines to $C$; if the characteristic of $k$ is two, then assume also that the $j$-invariant of $C$ is non-zero. The linear system $\left|-2 K_{S_{C}}\right|$ on $S_{C}$ is a base-point free pencil and the associated morphism $S_{C} \rightarrow \mathbb{P}^{1}$ is a rational elliptic surface with a unique multiple fiber corresponding to twice the unique element of $\left|-K_{S_{C}}\right|$.

Proof. By Lemma 4.2 the linear system $\left|-2 K_{S_{C}}\right|$ is base-point free and contains a connected and reduced divisor $R$. Applying Lemma 4.1 with $X=S_{C}$ and $N=R$, we deduce that the dimension of the linear system $\left|-2 K_{S_{C}}\right|$ is one and that the dimension of the linear system $\left|-K_{S_{C}}\right|$ is zero. The result follows.

Theorem 4.5: Let $C \subset \mathbb{P}^{2}$ be a smooth plane cubic curve over a field $k$ of characteristic relatively prime to six and let $T_{C} \subset\left(\mathbb{P}^{2}\right)^{\vee}$ be the set of inflection lines of $C$. If a plane sextic $\left(\mathbb{P}^{2}\right)^{\vee}$ is reduced, singular at all the points in $T_{C}$ and has a cusp at one of the points in $T_{C}$, then it is the dual curve $C^{\vee}$ of $C$ and, in particular, all of its singular points are cusps.

Proof. As before, denote by $S_{C}$ the blow up of $\left(\mathbb{P}^{2}\right)^{\vee}$ at the points of $T_{C}$, so that $S_{C}$ is a smooth projective rational surface. By Corollary 4.4, the linear system $\left|-2 K_{S_{C}}\right|$ induces a morphism $\varphi: S_{C} \rightarrow \mathbb{P}^{1}$ exhibiting $S_{C}$ as a rational elliptic surface with a multiple fiber $B$. One of the fibers of $\varphi$ corresponds to the sextic curve $C^{\vee} \subset\left(\mathbb{P}^{2}\right)^{\vee}$ dual to the curve $C$ : we denote this fiber of $\varphi$ by $\bar{C}^{\vee}$.

Let $f \in T_{C}$ be a point corresponding to an inflection line to $C$. Denote by $E_{f}$ the exceptional curve of $S_{C}$ lying above the point $f$, so that $\left(E_{f}\right)^{2}=$ $K_{S_{C}} \cdot E_{f}=-1$. A reduced curve $D$ in $\left|-2 K_{S_{C}}\right|$ intersects the exceptional curve $E_{f}$ at a subscheme of length two that is non-reduced exactly when the plane sextic corresponding to $D$ has a cusp at $f$. Moreover, the intersection of $E_{f}$ with a fiber of $\varphi$ is non-reduced precisely when the morphism $\left.\varphi\right|_{E_{f}}$ ramifies. The restriction of the morphism $\varphi$ to the curve $E_{f}$ has degree $\left(-2 K_{S_{C}}\right) \cdot E_{f}=2$, and hence it has exactly two ramification points. One of the ramification points is $E_{f} \cap B$, since the intersection of $E_{f}$ with the multiple fiber is non-reduced. We also know that the morphism $\left.\varphi\right|_{E_{f}}$ ramifies at the point $E_{f} \cap \bar{C}^{\vee}$, since the sextic corresponding to $\bar{C}^{\vee}$ is the dual of $C$ and hence it has a ! cusp at $f$. We therefore see that the two ramification points of the morphism $\left.\varphi\right|_{E_{f}}$ are one in 
the multiple fiber $2 B$ and the other in the fiber $\bar{C}^{\vee}$. Since this is true for all points $f \in T_{C}$, we conclude that the only reduced plane sextic having a singular point at each point of $T_{C}$ one of which is a cusp is the sextic $C^{\vee}$, and we are done.

Theorem 4.6 (Reconstruction Theorem): Let $C \subset \mathbb{P}^{2}$ be a smooth plane cubic curve over a field $k$ of characteristic different from three and let $T_{C} \subset\left(\mathbb{P}^{2}\right)^{\vee}$ be the set of inflection lines of $C$. There is a unique (geometrically) integral curve $C^{\prime} \subset\left(\mathbb{P}^{2}\right)^{\vee}$ such that:

- if $\operatorname{char}(k) \neq 2$, then $C^{\prime}$ is a sextic with cusps at the points of $T_{C}$;

- if $\operatorname{char}(k)=2$ and $j(C) \neq 0$, then $C^{\prime}$ is a cubic containing $T_{C}$;

- if $\operatorname{char}(k)=2$ and $j(C)=0$, then $C^{\prime}$ is a cubic containing $T_{C}$, with vanishing $j$-invariant.

Moreover, the space of cubics in $\left(\mathbb{P}^{2}\right)^{\vee}$ containing $T_{C}$ has dimension one if and only if $(\operatorname{char}(k), j(C))=(2,0)$. In all cases, the curve $C^{\prime}$ described above is the dual of the curve $C$.

Proof. If the characteristic of the field $k$ is different from two, then the result is a consequence of Theorem 4.5 ,

Suppose now that the characteristic of the field $k$ is two. In this case, the Gauss map is purely inseparable and the reduced image $C^{\vee}$ of the Gauss map of the curve $C$ is a cubic in $\left(\mathbb{P}^{2}\right)^{\vee}$ containing $T_{C}$. If the $j$-invariant of the curve $C$ is non-zero, then Corollary 4.4 implies that there is a unique cubic $C^{\prime}$ in $\left(\mathbb{P}^{2}\right)^{\vee}$ containing $T_{C}$, and hence the cubic $C^{\prime}$ must be the curve $C^{\vee}$, as required.

Finally, suppose that the $j$-invariant of the curve $C$ is zero (and $\operatorname{char}(k)=2$ ), so that the linear system $\Lambda$ of cubics in $\left(\mathbb{P}^{2}\right)^{\vee}$ containing $T_{C}$ has dimension at least one. The reduced dual $C^{\vee}$ of $C$ is an integral cubic in $\left(\mathbb{P}^{2}\right)^{\vee}$ containing $T_{C}$; Lemma 4.1 shows that the linear system $\Lambda$ has dimension exactly one. Since $\Lambda$ is a pencil of plane cubics containing smooth fibers, we deduce that the $j$-invariant of the pencil is a morphism of degree twelve. Since the curve $C$ has $j$-invariant zero and the characteristic of the field $k$ is two, the automorphism group of the curve $C$ has order 24 over any algebraically closed extension of $k$. In particular, the multiplicity of the fiber of the $j$-invariant corresponding to the curve $C^{\vee}$ is twelve, and hence there is a unique curve with $j$-invariant zero in the pencil $\Lambda$, namely $C^{\vee}$. Thus also in this case, the curve $C^{\prime}$ ! coincides with the curve $C^{\vee}$, and the result follows. 
AcKnowledgments. We want to thank E. Esteves for fundamental suggestions, and also A. Abreu, L. Caporaso, L. Gatto, D. Lehavi, N. Medeiros, E. Sernesi for precious discussions and I. Dolgachev for pointing out the reference [W]. We also thank the anonymous referee for his detailed report and for the constructive comments.

\section{References}

[AF] P. Aluffi and C. Faber, Linear orbits of arbitrary plane curves, The Michigan Mathematical Journal 48 (2000), 1-37.

[ACGH] E. Arbarello, M. Cornalba, P. A. Griffiths and J. Harris, Geometry of Algebraic Curves, Volume 1, Grundlehren der Mathematischen Wissenschaften, Vol. 267, Spinger-Verlag, New York, 1985.

[AD] M. Artebani and I. Dolgachev, The Hesse pencil of plane cubic curves, L'Enseignement Mathématique. Revue Internationale. 2e Série 55 (2009), 235-273.

[BKT] F. Bogomolov, M. Korotiaev and Y. Tschinkel, A Torelli theorem for curves over finite fields Pure and Applied Mathematics Quarterly 6 (2010), 245-294.

[CS1] L. Caporaso and E. Sernesi, Recovering plane curves from their bitangents, Journal of Algebraic Geometry 12 (2003), 225-244.

[CS2] L. Caporaso and E. Sernesi, Characterizing curves by their odd theta-characteristics, Journal für die Reine und Angewandte Mathematik 562 (2003), 101-135.

[EH1] D. Eisenbud and J. Harris, Limit linear series: Basic theory, Inventiones Mathematicae 85 (1986), 337-371.

[EH2] D. Eisenbud and J. Harris, The Geometry of Schemes, Graduate Texts in Mathematics 197, Springer, Berlin, 2000.

[EM] E. Esteves and N. Medeiros, Limit canonical systems on curves with two components, Inventiones Mathematicae 149 (2002), 267-338.

[G] A. Grothendieck, Élements de géométrie algébrique III, Institut des Hautes Études Scientifiques. Publications Mathématiques 11 (1961), 5-167.

[GS] S. Grushevsky and R. Salvati Manni, Gradients of odd theta functions, Journal für die Reine und Angewandte Mathematik 573 (2004), 45-59.

[HM] J. Harris and I. Morrison, Moduli of Curves, Graduate Texts in Mathematics, Vol. 187, Springer-Verlag, New York, 1998.

[H1] O. Hesse, Über die Elimination der Variabeln aus drei algebraischen Gleichungen vom zweiten Grade mit zwei Variabeln, Journal für die Reine und Angewandte Mathematik 28 (1844), 68-96.

[H2] O. Hesse, Über die Wendepunkte der Curven dritter Ordnung, Journal für die Reine und Angewandte Mathematik 28 (1844), 97-102.

[K1] S. Katz, Tangents to a multiple plane curve, Pacific Journal of Mathematics 124 (1986), 321-331.

[K2] S. Katz, Discriminant and limits of duals of plane curves, in Enumerative Algebraic Geometry, Proceedings of the 1989 Zeuthen Symposium, Contemporary Mathematics 123, American Mathematical Society, Providence, RI, 1991. 
[La] R. Lax, On the distribution of Weierstrass points on singular curves, Israel Journal of of Mathematics $5 \mathbf{7}$ (1987), 107-115.

[L1] D. Lehavi, Any smooth plane quartic can be reconstructed from its bitangents, Israel Journal of Mathematics 146 (2005), 371-379.

[L2] D. Lehavi, Effective reconstruction of curves from their theta hyperplanes, available at http://arxiv.org/abs/1001.1237

[MFK] D. Mumford, J. Forgarty and F. Kirwan, Geometric Invariant Theory, Ergebnisse der Mathematik und ihrer Grenzgebiete (2) 34, Springer-Verlag, Berlin, 1994.

[N] P. E. Newstead, Introduction to Moduli Problems and Orbit Spaces, Tata Institute of Fundamental Research, Bombay, Springer-Verlag, Berlin, 1978.

[P] M. Pacini, Spin curves over non stable curves, Communications in Algebra 36 (2008), 1365-1393.

[WL] C. Widland and R. Lax, Weierstrass Points on Gorenstein Curves, Pacific Journal of Mathematics 142 (1990), 197-208.

[W] R. M. Winger, On the invariants of the ternary icosahedral group, Mathematische Annalen 93 (1925), 210-216. 